Nonlinear Processes in Geophysics, 12, 407-423, 2005

SRef-ID: $1607-7946 / \mathrm{npg} / 2005-12-407$

European Geosciences Union

(C) 2005 Author(s). This work is licensed

under a Creative Commons License.

\title{
Exact theory for localized envelope modulated electrostatic wavepackets in space and dusty plasmas
}

\author{
I. Kourakis ${ }^{1,2}$ and P. K. Shukla ${ }^{1}$ \\ ${ }^{1}$ Institut für Theoretische Physik IV, Fakultät für Physik und Astronomie, Ruhr-Universität Bochum, D-44780 Bochum, \\ Germany \\ ${ }^{2}$ Max-Planck-Institut für extraterrestrische Physik, Giessenbachstrasse, D-85740 Garching, Germany
}

Received: 2 November 2004 - Accepted: 25 January 2005 - Published: 18 March 2005

Part of Special Issue "Nonlinear plasma waves-solitons, periodic waves and oscillations"

\begin{abstract}
Abundant evidence for the occurrence of modulated envelope plasma wave packets is provided by recent satellite missions. These excitations are characterized by a slowly varying localized envelope structure, embedding the fast carrier wave, which appears to be the result of strong modulation of the wave amplitude. This modulation may be due to parametric interactions between different modes or, simply, to the nonlinear (self-)interaction of the carrier wave.

A generic exact theory is presented in this study, for the nonlinear self-modulation of known electrostatic plasma modes, by employing a collisionless fluid model. Both cold (zero-temperature) and warm fluid descriptions are discussed and the results are compared. The (moderately) nonlinear oscillation regime is investigated by applying a multiple scale technique. The calculation leads to a Nonlinear Schrödingertype Equation (NLSE), which describes the evolution of the slowly varying wave amplitude in time and space. The NLSE admits localized envelope (solitary wave) solutions of bright(pulses) or dark- (holes, voids) type, whose characteristics (maximum amplitude, width) depend on intrinsic plasma parameters. Effects like amplitude perturbation obliqueness (with respect to the propagation direction), finite temperature and defect (dust) concentration are explicitly considered. Relevance with similar highly localized modulated wave structures observed during recent satellite missions is discussed.
\end{abstract}

\section{Introduction}

In a wide variety of physical contexts, the dynamics of propagating periodic excitations (waves) is dominated by a competition between the effects of "mode dispersion" and "nonlinearity" of the medium. The latter mechanism, which is ignored when studying harmonic (linear) wave propagation in the small-amplitude limit, generally increases when the dis-

Correspondence to: I. Kourakis

(ioannis@ tp4.rub.de) placement from equilibrium grows bigger. For a sufficiently important excitation amplitude, it is known that nonlinearity may be strong enough to balance spatial delocalization (i.e. mode separation due to dispersion) and thus result to the formation of propagating localized structures (solitary waves, solitons). On the other hand, weakly nonlinear effects enter into play as the wave amplitude acquires (even small yet) finite (non negligible) values, moderately beyond the linear approximation. The generic signature of this mechanism is the appearance of secondary phase "harmonics" in the Fourier spectrum of the system observed, in addition to a nonlinear modulation of the wave's amplitude, manifested as a slow variation of the wave's amplitude in space and time. The occurrence of amplitude modulation may be due to parametric wave coupling, interaction between high- and low- frequency modes or, simply, self-interaction of the carrier wave ("auto"or "self"-modulation). Furthermore, analytical and numerical studies have established the relevance of these phenomena with modulational instability, which may lead to energy localization via localized pulse formation, as known in fields as diverse as Nonlinear Optics, Condensed Matter Physics and Biophysics (Davydov, 1985; Hasegawa, 1989; Infeld, 1990; Remoissenet, 1994).

Charged matter (plasma), a nonlinear and dispersive medium "par excellence", provides a typical paradigm for the study of such mechanisms. As far as plasma modes are concerned (Krall and Trivelpiece, 1973; Stix, 1992), the occurrence of such phenomena has been confirmed by experiments related to the nonlinear propagation of electrostatic (ES, e.g. ion-acoustic) (Watanabe 1977; Bailung and Nakamura, 1993; Luo et al., 1998; Nakamura et al., 1999; Nakamura and Sarma, 2001) as well as electromagnetic (EM, e.g. whistler) waves (Kostrov, 2003). Recent numerical simulations of electron cyclotron waves (Eliasson and Shukla, 2004) (as well as earlier ones, by Hasegawa, 1970, 1972) also predict such a behaviour.

In the context of Space Physics, moving localized electrostatic structures have been reported by recent spacecraft missions e.g. the FAST at the auroral region (Delory et al., 1998; 


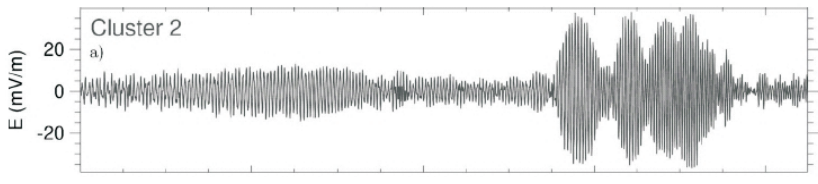

Fig. 1. Modulated structures, related to "chorus" (EM) emission in the magnetosphere (CLUSTER satellite data; reprinted from Santolik, 2003).

Ergun et al., 1998a, 1998b; Pottelette et al., 1999), as well as the S3-3 (Temerin, 1982), Viking (Boström, 1988), GEOTAIL and POLAR earlier missions in the magnetosphere (Matsumoto et al., 1994; Franz et al., 1998; Cattell et al., 1999, 2003; McFadden et al., 2003) (also see many references therein) (the interpretation of the Viking measurements, Bostrom, 1988, has recently risen some doubt; see the thorough discussion in McFadden et al., 2003). Some of the localized structures reported therein bear qualitative characteristics which are reminiscent of solitary electrostatic waves and are strongly believed to be related to ion acoustic waves; see the discussion in (McFadden et al., 2003). It should be stressed that both compressive and rarefactive large amplitude structures have been observed (Matsumoto et al., 1994; Franz et al., 1998; Cattell et al., 1999, 2003) (also see many references therein). Note that it was recently suggested by McFadden et al. (2003) that neither the velocity dependence of the observed potential structure amplitudes nor their asymmetry should be taken for granted, since they may be attributed to intrinsic measurement errors. Finally, the observed phase speeds lie over an extended region of values, sometimes even above the ion sound velocity; these facts seem to suggest that plainly employing the soliton (Korteweg - deVries, KdV) picture may not suffice for the elucidation of the generation of these solitary structures and an alternative instability mechanism may be present; also see the discussion in (Berthomier et al., 1998; McFadden et al., 2003). Localized modulated wave packets, in particular, are encountered in abundance e.g. in the Earth's magnetosphere, where they are associated with localized field and/or density variations simultaneously observed (Pottelette et al., 1999; Alpert, 2001; Santolik, 2003). The occurrence of such wave forms is, for instance, thought to be related to the broadband electrostatic noise (BEN) encountered in the "auroral" region (Pottelette et al., 1999).

Recent analytical studies have supplied evidence for the relevance of nonlinear modulational effects in dustcontaminated plasmas ("Dusty" or "Complex" Plasmas), where a strong presence of mesoscopic, massive, charged dust grains strongly affects the characteristics of the plasma (Verheest, 2001; Shukla and Mamun, 2002). The modification of the plasma response due to the presence of dust gives rise to new ES/EM modes, whose self-modulation was recently shown to lead to modulational instability and soliton formation; these include e.g. the dust-acoustic (DA) (Rao et al., 1990; Amin et al., 1998; Tang and Xue, 2003; Kourakis

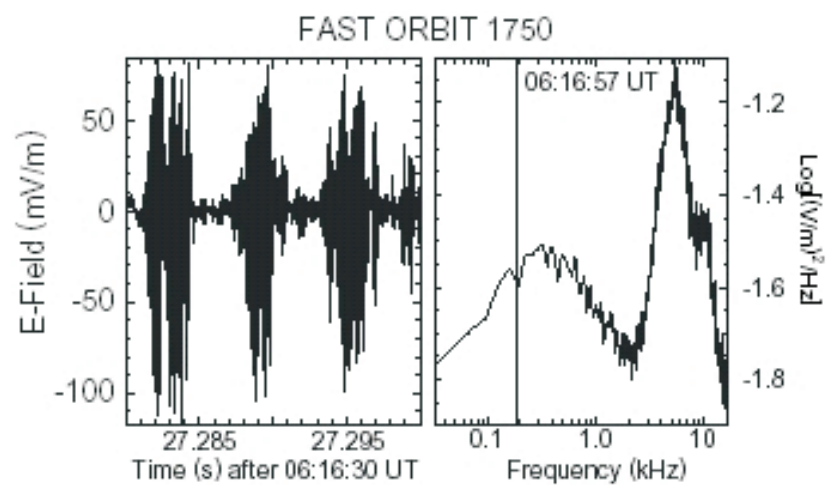

Fig. 2. Electrostatic noise wave forms, related to modulated electron-acoustic waves (FAST satellite data; figure reprinted from Pottelette et al., 1999). The co-existence of a high (carrier) and a low (modulated envelope) frequencies is clearly reflected in the Fourier spectrum, in the right.

and Shukla, 2004a) and dust-ion acoustic (DIA) ES modes (Shukla and Silin, 1992; Amin et al., 1998; Kourakis and Shukla, 2003a, 2004b), in addition to magnetized plasma modes, e.g. the Rao EM dust mode (Kourakis and Shukla, 2004c).

The purpose of this paper is to provide a "generic" methodological framework for the study of the nonlinear (self-) modulation of the amplitude of electrostatic (ES) plasma modes. The results which follow cover a variety of ES modes. We mean to emphasize the generic character of the nonlinear behaviour of these modes, so focusing upon a specific mode is avoided on purpose. Where appropriate, details regarding specific modes may be sought in (Kourakis and Shukla, 2003a, b, 2004a, b, d), where some of this material was first presented.

In the following, we study the modulational instability of electrostatic plasma waves propagating "along" the magnetic field, so that the Lorentz forces can be omitted. Amplitude modulation is allowed to take place in an oblique direction, at an angle $\theta$ with respect to the carrier wave propagation direction. By assuming the wave's amplitude to vary on slow space and time scales, say $X$ and $T$ (see definitions below), we shall seek an evolution equation for the amplitude $\psi(X, T)$, establish its oscillatory solution and then establish an explicit criterion for modulational (in)stability. Our aim is to trace the influence of $\theta$ on the conditions for modulational instability onset, and determine the magnitude of the associated instability growth rate. We shall also examine the possibility of the formation of localized excitations and discuss their characteristics. Exact new expressions are derived for quantities of interest, in terms of the system's dispersion laws and the intrinsic plasma parameters.

The manuscript is organized as follows. In the next Section, the analytical model is introduced. In Sect. 3, we carry out a perturbative analysis by introducing appropriate slow space and time evolution scales, and derive a NLS-type equation which governs the (slow) amplitude evolution in time 


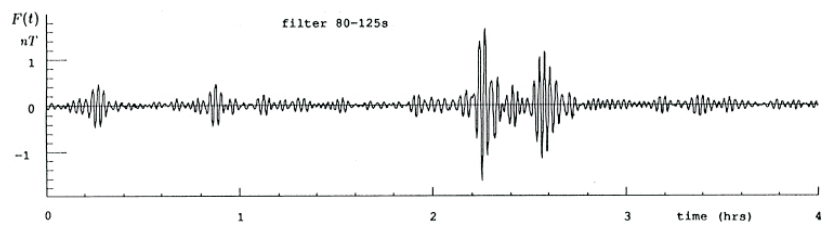

Fig. 3. Localized envelope structures in the magnetosphere reprinted from Alpert (2001).

and space. The exact form of dispersion and nonlinearity coefficients in the NLS-type equation is presented and discussed. In Sect. 4, we carry out a stability analysis of the NLSE allowing for a thorough study of the DA wave stability in various regions of the physical parameters involved. We pursue the analysis in Sect. 5, by discussing the possibility of the existence of localized solutions of the NLSE, and identifying their forms in different parameter regions. The relevance of the formalism with known plasma modes is discussed in Sect. 6. Finally, our results are briefly discussed and summarized in the concluding Sect. 7.

\section{The model: formulation and analysis}

In a general manner, several known electrostatic plasma modes (Stix, 1992; Swanson, 2003) are adequately described by (single) fluid models; ES plasma modes are thus associated with propagating oscillations which are related to "one" dynamical plasma constituent, say $\alpha$ (mass $m_{\alpha}$, charge $q_{\alpha} \equiv s_{\alpha} Z_{\alpha} e ; e$ is the absolute electron charge; $s=s_{\alpha}=$ $q_{\alpha} /\left|q_{\alpha}\right|= \pm 1$ is the charge "sign"), against a background of one (or more) constituent(s), say $\alpha^{\prime}$ (mass $m_{\alpha^{\prime}}$, charge $q_{\alpha^{\prime}} \equiv$ $s_{\alpha^{\prime}} Z_{\alpha^{\prime}} e$, similarly). The background species is (are) often assumed to obey a known distribution, e.g. to be in a fixed (uniform) or in a thermalized (Maxwellian) state, for simplicity, depending on the particular aspects (e.g. frequency scales) of the physical system considered. For instance, the "ionacoustic" (IA) mode refers to ions $(\alpha=i)$ oscillating against a (much hotter) electron background $\left(\alpha^{\prime}=e\right)$, which may be considered to be Maxwellian (Krall and Trivelpiece, 1973; Kourakis and Shukla, 2003b); the "electron-acoustic" (EA) mode (Krall and Trivelpiece, 1973; Kourakis and Shukla, 2004d) can be modelled as electron oscillations $(\alpha=e)$ against a background of ions $\left(\alpha^{\prime}=i\right)$, which are practically immobile (fixed), and so forth (Krall and Trivelpiece, 1973; Stix, 1992). The coexistence of "hot" $(h)$ and "cold" $(c)$ electron populations, observed in the upper parts of the Earth's magnetosphere (Berthomier et al., 1998), may also readily be accommodated in this description, in order to study its influence on IA $\left(\alpha=i, \alpha^{\prime}=c, h\right)$ (Kourakis and Shukla, 2003b) and EA ( $\left.\alpha=c, \alpha^{\prime}=i, h\right)$ (Kourakis and Shukla, 2004d) waves. As regards "dusty plasma" modes, the DA mode describes oscillations of dust grains $(\alpha=d)$ against a Maxwellian electron and ion background $\left(\alpha^{\prime}=e, i\right)$ (Shukla and Mamun, 2002; Kourakis and Shukla, 2004a), while DIA waves denote IA oscillations in the presence of inertial dust in the background $\left(\alpha=i, \alpha^{\prime}=e, d\right.$ ) (Shukla and Mamun, 2002; Kourakis and Shukla, 2003a; Kourakis and Shukla, 2004b).

\subsection{A generic fluid description}

A standard (single) fluid model to be employed for the dynamic species $\alpha$ consists of the first moment evolution equations, namely the density $n_{\alpha}$ (conservation) equation, the mean fluid velocity equation and the pressure equation:

$$
\begin{aligned}
& \frac{\partial n_{\alpha}}{\partial t}+\nabla \cdot\left(n_{\alpha} \mathbf{u}_{\alpha}\right)=0 \\
& \frac{\partial \mathbf{u}_{\alpha}}{\partial t}+\mathbf{u}_{\alpha} \cdot \nabla \mathbf{u}_{\alpha}=-\frac{q_{\alpha}}{m_{\alpha}} \nabla \Phi-\frac{1}{m_{\alpha} n_{\alpha}} \nabla p_{\alpha} \\
& \frac{\partial p_{\alpha}}{\partial t}+\mathbf{u}_{\alpha} \cdot \nabla p_{\alpha}=-\gamma p_{\alpha} \nabla \cdot \mathbf{u}_{\alpha},
\end{aligned}
$$

where $n_{\alpha}, \mathbf{u}_{\alpha}$ and $p_{\alpha}$ respectively denote the "density", "mean" (fluid) "velocity" and "pressure" of species $\alpha$. The parameter $\gamma=c_{P} / c_{V}=1+2 / f$ denotes the specific heat ratio (for $f$ degrees of freedom), e.g. $\gamma=3$ in the one-dimensional ("1d") case, $\gamma=2$ in the two-dimensional (" $2 \mathrm{~d} ")$ and $\gamma=5 / 3$ in the three-dimensional ("3d") case; also, $\gamma=1$ if an adiabatic evolution is considered.

The electric potential $\Phi$ obeys Poisson's eq.:

$$
\nabla^{2} \Phi=-4 \pi \sum_{\alpha^{\prime \prime}=\alpha,\left\{\alpha^{\prime}\right\}} q_{\alpha^{\prime \prime}} n_{\alpha^{\prime \prime}}=4 \pi e\left(n_{e}-Z_{i} n_{i}+\ldots\right),
$$

where all the particle species appear in the right-hand side ("rhs"). Overall charge neutrality is assumed at equilibrium, i.e.

$q_{\alpha} n_{\alpha, 0}=-\sum_{\alpha^{\prime}} q_{\alpha^{\prime}} n_{\alpha^{\prime}, 0}$.

\subsection{Reduced description}

By choosing appropriate scales for all quantities, the above system of evolution equations may be cast into the following form:

$$
\begin{aligned}
& \frac{\partial n}{\partial t}+\nabla \cdot(n \mathbf{u})=0, \\
& \frac{\partial \mathbf{u}}{\partial t}+\mathbf{u} \cdot \nabla \mathbf{u}=-s \nabla \phi-\frac{\sigma}{n} \nabla p, \\
& \frac{\partial p}{\partial t}+\mathbf{u} \cdot \nabla p=-\gamma p \nabla \cdot \mathbf{u}
\end{aligned}
$$

(the index $\alpha$ will be understood where omitted, viz. $s=s_{\alpha}$ ). The re-scaled (dimensionless) dynamic variables are now: $n=n_{\alpha} / n_{\alpha, 0}, \mathbf{u}=\mathbf{u}_{\alpha} / c_{*}, p=p_{\alpha} /\left(n_{\alpha, 0} k_{B} T_{\alpha}\right)$, and $\phi=\left|q_{\alpha}\right| \Phi /\left(k_{B} T_{*}\right)$, where $n_{\alpha, 0}$ is the equilibrium density and $c_{*}=\left(k_{B} T_{*} / m_{\alpha}\right)^{1 / 2}$ is a characteristic (e.g. sound) velocity. Time and space are scaled over appropriately chosen scales $t_{0}$ (e.g. $\left.\omega_{p, \alpha}^{-1}=\left(4 \pi n_{\alpha, 0} q_{\alpha}^{2} / m_{\alpha}\right)^{-1 / 2}\right)$ and $r_{0}=c_{*} t_{0}$; $T_{\alpha}$ is the fluid temperature (so pressure at equilibrium is: 
$p_{0}=n_{\alpha, 0} k_{B} T_{\alpha}$ ), and $T_{*}$ is an effective temperature (related to the background considered), to be determined for each problem under consideration ( $k_{B}$ is Boltzmann's constant). The temperature ratio $T_{\alpha} / T_{*}$ is denoted by $\sigma$, in this "warm model" (Chan and Seshadri, 1975; Durrani et al., 1979) (the so-called "cold model" is recovered for $\sigma=0$; see that Eq. (6) then becomes obsolete). The Lorentz force term was omitted, since wave propagation along the external magnetic field is considered here. The system is closed by Poisson's equation, which may now be expressed as ${ }^{1}$

$\nabla^{2} \phi=-s\left[n+\sum_{\alpha^{\prime}} n_{\alpha^{\prime}} q_{\alpha^{\prime}} /\left(n_{\alpha, 0} q_{\alpha}\right)\right] \equiv-s(n-\hat{n})$.

Note that the neutralizing background (reduced) density

$\hat{n}=-\sum_{\alpha^{\prime}} \frac{n_{\alpha^{\prime}} q_{\alpha^{\prime}}}{n_{\alpha, 0} q_{\alpha}}=-\frac{1}{s_{\alpha} Z_{\alpha} n_{\alpha, 0}} \sum_{\alpha^{\prime}} s_{\alpha^{\prime}} Z_{\alpha^{\prime}} n_{\alpha^{\prime}}$

is a priori ${ }^{2}$ a function of the potential $\phi$ (note that $\hat{n}=1$ for $\phi=0$, due to the equilibrium neutrality condition); furthermore, it depends on the physical parameters (e.g. background temperature, plasma density, defect concentration, ...) involved in a given problem. The calculation in the specific case of IA waves is explicitly provided below, for clarity.

\subsection{Weakly nonlinear oscillation regime}

What follows is essentially an implementation of the long known "reductive perturbation" technique (Taniuti and Yajima, 1969; Asano et al., 1969; Shimizu and Ichikawa, 1972; Kako, 1974; Kakutani, 1974), which was first applied in the study of electron plasma (Taniuti and Yajima, 1969; Asano et al., 1969) and electron-cyclotron (Hasegawa 1970, 1972) waves, more than three decades ago.

Equations (4)-(7) describe the evolution of the state vec$\operatorname{tor}^{3} \boldsymbol{S}=\{n, \mathbf{u}, p, \phi\}$ which accepts a harmonic (electrostatic) wave solution in the form $\mathbf{S}=\mathbf{S}_{\mathbf{0}} \exp [i(\mathbf{k r}-\omega \mathbf{t})]+$ c.c., in the weak amplitude approximation, i.e. for $S_{0, j} \ll 1$. Once the amplitude of this wave becomes non-negligible, a nonlinear harmonic generation mechanism enters into play: this is the first signature of nonlinearity, which manifests its presence once a slight departure from the weak-amplitude (linear) domain occurs. In order to study the nonlinear (amplitude) modulational stability profile of these electrostatic waves, we consider small deviations from the equilibrium state $\mathbf{S}^{(0)}=(1, \mathbf{0}, 1,0)^{T}$, viz. $\mathbf{S}=\mathbf{S}^{(0)}+\epsilon \mathbf{S}^{(1)}+\epsilon^{2} \mathbf{S}^{(2)}+\ldots$, where $\epsilon \ll 1$ is a smallness parameter. We have assumed that ${ }^{4} S_{j}^{(n)}=\sum_{l=-\infty}^{\infty} S_{j, l}^{(n)}(X, T) \exp [i l(\mathbf{k r}-\omega \mathbf{t})]$

\footnotetext{
${ }^{1} \mathrm{~A}$ factor $\omega_{p, \alpha}^{2} t_{0}^{2}$ is omitted in the right-hand side of Eq. (7), since equal to 1 for $t_{0}=\omega_{p, \alpha}^{-1}$.

${ }^{2}$ This is only not true when the background is assumed fixed, e.g. for EA waves (i.e. $s_{\alpha}=-s_{\alpha^{\prime}}=-1, n_{\alpha^{\prime}}=n_{i}=$ const.), where $\hat{n}=Z_{i} n_{i} / n_{e, 0}=$ const.

${ }^{3}$ Note that $S \in \Re^{d+3}$ in a $d$-dimensional problem $(d=$ $1,2,3)$.

${ }^{4}$ In practice, only terms with $l \leq n$ do contribute in this summation. This simply means that up to 1 st harmonics are expected for $n=1$, up to 2 nd phase harmonics for $n=2$, and so forth.
}

(for $j=1,2, \ldots, d+3$; see footnote 3 ; the condition $S_{j,-l}^{(n)}=S_{j, l}^{(n)^{*}}$ holds, for reality). The wave amplitude is thus allowed to depend on the stretched ("slow") coordinates $X=\epsilon(x-\lambda) t$ and $T=\epsilon^{2} t$; the real variable $\lambda$, to be determined, will later be interpreted as the wave's "group velocity" along the modulation direction $x$. The amplitude modulation direction $(\sim \hat{x})$ is assumed "oblique" with respect to the (arbitrary) propagation direction, which is expressed by the wave vector $\boldsymbol{k}=\left(k_{x}, k_{y}\right)=(k \cos \theta, k \sin \theta)$; cf. (Kako and Hasegawa, 1976; Chhabra and Sharma, 1986; Mishra et al., 1994), where a similar treatment is adopted. Note that (not having taken the magnetic field into account in the analysis) this is essentially a " $2 \mathrm{~d}$ " physical problem, although readily applicable in a three-dimensional ("3d") description, for completeness. We shall limit ourselves to considering two axes ( $x$ and $y$ ) in the following.

According to the above considerations, we set:

$\partial / \partial t \rightarrow \partial / \partial t-\epsilon \lambda \partial / \partial X+\epsilon^{2} \partial / \partial T$,

$\partial / \partial x \rightarrow \partial / \partial x+\epsilon \partial / \partial X$

(while $\partial / \partial y$ remains unchanged) and

$\nabla^{2} \rightarrow \nabla^{2}+2 \epsilon \partial^{2} / \partial x \partial X+\epsilon^{2} \partial^{2} / \partial X^{2}$,

so that

$$
\begin{aligned}
& \frac{\partial}{\partial t} A_{l}^{(n)} e^{i l \theta_{1}}=\left(-i l \omega A_{l}^{(n)}-\epsilon \lambda \frac{\partial A_{l}^{(n)}}{\partial X}+\epsilon^{2} \frac{\partial A_{l}^{(n)}}{\partial T}\right) \\
& \times e^{i l \theta_{l}}\left(i l \mathbf{k} A_{l}^{(n)}+\epsilon \hat{x} \frac{\partial A_{l}^{(n)}}{\partial X}\right) e^{i l \theta_{1}}, \\
& \nabla_{l}^{2} A_{l}^{(n)} e^{i l \theta_{1}}=\left(-l^{2} k^{2} A_{l}^{(n)}+2 \epsilon i l k_{x} \frac{\partial A_{l}^{(n)}}{\partial X}\right. \\
&\left.+\epsilon^{2} \frac{\partial^{2} A_{l}^{(n)}}{\partial X^{2}}\right) e^{i l \theta_{1}}
\end{aligned}
$$

for any $l$-th phase harmonic amplitude $A_{l}^{(n)}$ among the components of $\mathbf{S}_{l}^{(n)}$; obviously, $\theta_{1}$ here denotes the elementary phase $\theta_{1} \equiv \mathbf{k r}-\omega t$.

By expanding near $\phi \approx 0$, Poisson's eq. may formally be cast in the form

$\nabla^{2} \phi=\phi-\alpha \phi^{2}+\alpha^{\prime} \phi^{3}-s \beta(n-1)$,

where the exact form of the real coefficients $\alpha, \alpha^{\prime}$ and $\beta$ (to be distinguished from the species indices above, obviously) are to be determined exactly for any specific problem, and contain all the essential dependence on the plasma parameters. Note that the right-hand side in Eq. (10) cancels at equilibrium.

The system of Eqs. (4)-(6) and (10) determines the evolution of the physical system considered, and is the basis of the analytical study which follows. 


\subsection{A case study: ion-acoustic waves}

In order to make our method and notation concise and clear, let us explicitly consider the simple case of "ions" (thus $\alpha=$ $i$ and $q_{\alpha}=q_{i}=+Z_{i}$ e, i.e. $\left.s_{\alpha}=+1\right)$ oscillating against thermalized "electrons" (viz. $\alpha^{\prime}=e$ and $q_{\alpha^{\prime}}=q_{e}=-e$, i.e. $\left.s_{\alpha^{\prime}}=-1 ; n_{e}=n_{e, 0} e^{e \Phi /\left(k_{B} T_{e}\right)}\right)$. Adopting the scaling defined above, and using the equilibrium neutrality condition $n_{e, 0}=Z_{i} n_{i, 0}$, it is a trivial exercise to cast Poisson's Eq. (2) into the (reduced) form:

$\nabla^{2} \phi=-\left(\omega_{p, i} r_{0} / c_{*}\right)^{2}\left[n-e^{T_{*} \phi /\left(Z_{i} T_{e}\right)}\right] \equiv-\left(n-e^{\xi \phi}\right)$,

where we took: $t_{0}=r_{0} / c_{*}=\omega_{p, i}^{-1}$ and $\xi \equiv T_{*} /\left(Z_{i} T_{e}\right)$. Now, expanding near $\phi \approx 0$, viz. $e^{\xi \phi} \approx 1+\xi \phi+\xi^{2} \phi^{2} / 2+$ $\xi^{3} \phi^{3} / 6+\ldots$, we have:

$\nabla^{2} \phi \approx \xi \phi+\xi^{2} \phi^{2} / 2+\xi^{3} \phi^{3} / 6-(n-1)$.

Finally, setting the temperature scale $T_{*}$ equal to $T_{*}=Z_{i} T_{e}$, for convenience (so that $\xi=1$ ) $^{5}$, one recovers exactly Eq. (10) with $\alpha=-1 / 2, \alpha^{\prime}=1 / 6$ and $\beta=1$.

The amplitude modulation of ion-acoustic waves was first studied for parallel modulation (i.e. in the direction of propagation, viz. $\theta=0$ ) in (Shimizu and Ichikawa, 1972), and for oblique modulation in (Kako and Hasegawa, 1976); as expected, those results are recovered from the formulae below.

\section{Perturbative analysis}

By substituting the perturbative series defined in the previous Section into Eqs. (4)-(6) and (10) and isolating distinct orders in $\epsilon$, we obtain a set of reduced equations for the harmonic amplitude at each ( $n$ th-) order. Solving at each order and substituting in th following one, one successively obtains a linear homogeneous (Cramer-type) system of equations, whose solution provides the amplitudes of the $l-$ th harmonic contributions at order $\epsilon^{n}$ (i.e. $n_{l}^{(n)}, u_{x / y, l}^{(n)}, p_{l}^{(n)}, \phi_{l}^{(n)}$, viz. $l=0,1,2, \ldots, n$ for every $n=1,2,3, \ldots)$. The compatibility condition obtained at each order, appearing e.g. as the requirement of a determinant to be equal to zero, need also be taken into account.

The details of the tedious calculation are explicitly provided in the Appendix, so only a few essential steps are exposed in the following, for clarity.

\subsection{1st-order quantities - dispersion relation}

The first harmonic amplitudes are determined (to order $\sim \epsilon^{1}$ ) as

$n_{1}^{(1)}=s \frac{1+k^{2}}{\beta} \psi=\frac{k}{\omega \cos \theta} u_{1, x}^{(1)}=\frac{k}{\omega \sin \theta} u_{1, y}^{(1)}=\frac{p_{1}^{(1)}}{\gamma}$

\footnotetext{
${ }^{5}$ Note that a different choice for $T_{*}$ would lead to a modified right-hand-side in Eq. (10), i.e. a factor $\xi \neq 1$ would precede the first term (in $\phi$ ). This might, of course, also be a legitimate choice of scaling; however, the following formula are not valid - and should be appropriately modified - in this case. Obviously though, the qualitative results of this study are not affected by the choice of scaling.
}

in terms e.g. of the potential correction $\phi_{1}^{(1)} \equiv \psi$, along with the dispersion relation

$$
\omega^{2}=\frac{\beta k^{2}}{k^{2}+1}+\gamma \sigma k^{2} .
$$

See that the expected acoustic behaviour $\omega \sim k$ is obtained for low wavelengths, i.e. for $k \ll 1$.

\subsection{2nd-order quantities - group velocity}

The amplitudes of the 2nd and 0th (constant) harmonic corrections are obtained in order $\sim \epsilon^{2}$ (the lengthy expressions can be found in the Appendix); retain that the former (later) are proportional to $\psi^{2}\left(|\psi|^{2}\right.$, respectively).

The compatibility condition obtained for $n=2$ and $l=1$ provides exactly the constraint:

$\lambda=\frac{\partial \omega(k)}{\partial k_{x}} \equiv v_{g}$,

suggesting that the parameter $\lambda$ denotes the velocity $v_{g}$ at which the wave's amplitude travels along the modulation direction $x$; for parallel modulation (viz. $\theta=0$ ) this is simply the "group velocity" $\partial \omega(k) / \partial k^{6}$; see Eq. (A12) in the Appendix for the exact expression for $v_{g}$.

\subsection{3rd-order quantities - the envelope evolution equation}

At this stage, one has obtained a solution up to second order, viz.

$n \approx n_{0}+\epsilon\left\{n_{1}^{(1)} \exp [i(\mathbf{k r}-\omega t)]+\right.$ c.c. $\}$

$+\epsilon^{2}\left\{n_{0}^{(2)}+\left\{n_{2}^{(2)} \exp [2 i(\mathbf{k r}-\omega t)]+\right.\right.$ c.c. $\left.\}\right\}+\mathcal{O}\left(\epsilon^{3}\right)$

for the density (see that we have taken $n_{1}^{(2)}=0$, with no loss of generality), along with analogous expressions for the other state variables, namely velocity components $u_{x / y}$, pressure $p$ and potential $\phi$.

Isolating the evolution equations for $n=3$ and $l=1$, one obtains an explicit condition for suppression of "secular terms" to be obeyed by the potential correction $\psi$, i.e. a compatibility condition in the form of a "nonlinear Schrödingertype equation" (NLSE)

$i \frac{\partial \psi}{\partial T}+P \frac{\partial^{2} \psi}{\partial X^{2}}+Q|\psi|^{2} \psi=0$.

\footnotetext{
${ }^{6}$ This is a - physically expected - constraint which is imposed by the equations for $n=2$ and $l=1$ (1st harmonics at 2nd order). Alternatively, one may assume a dependence on $X_{n}=\epsilon^{n} x$ (plus a similar expansion for $y, z$ and $t$ ) for $n=0,1,2, \ldots$; the condition for annihilation of secular terms then reads: $\partial A_{1}^{(1)} / \partial T_{1}+$ $\left(\partial \omega / \partial k_{x}\right) \partial A_{1}^{(1)} / \partial X_{1}$, i.e. $A_{1}^{(1)}=A_{1}^{(1)}\left(X_{1}-v_{g} T_{1}\right)$ (for any of the 1 st harmonic amplitudes $\left.A_{1}^{(1)} \in\left\{S_{1, j}^{(1)}\right\}\right)$, which essentially amounts to the same constraint
} 


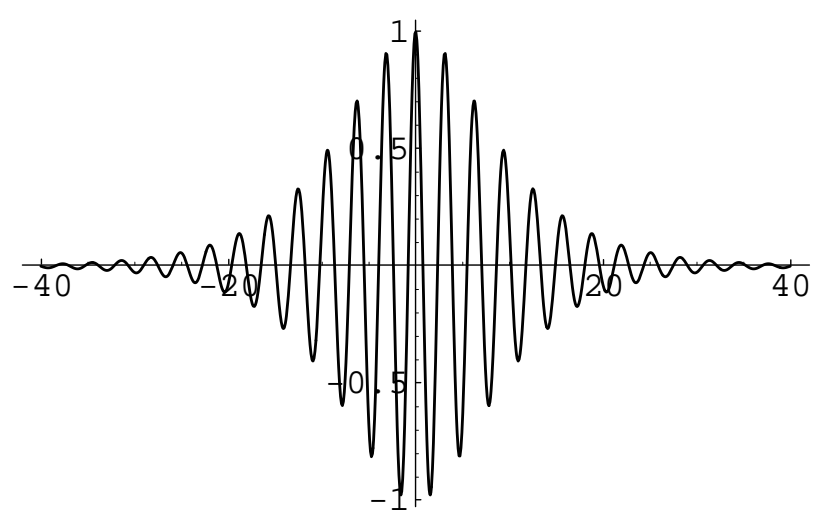

(a)

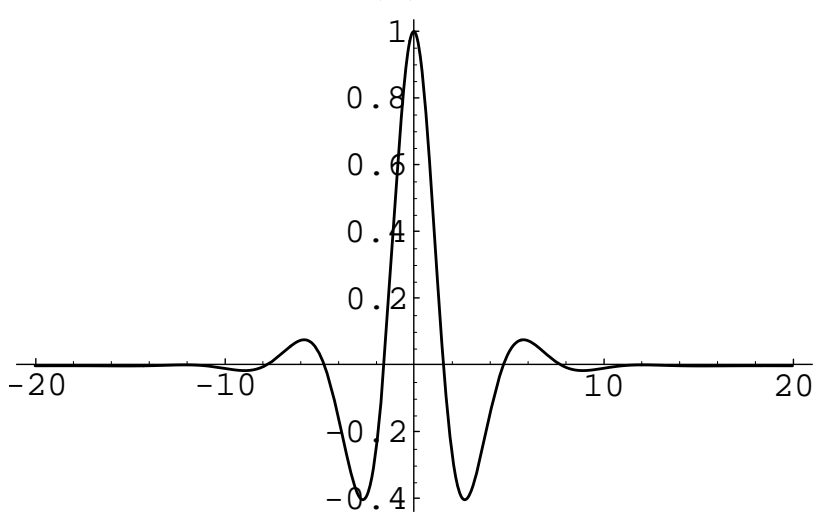

(b)

Fig. 4. "Bright" type modulated wavepackets (for $P Q>0$ ), for two different (arbitrary) sets of parameter values.

The "dispersion coefficient" $P$ is in fact related to the curvature of the dispersion curve as

$P=\frac{1}{2} \frac{\partial^{2} \omega}{\partial k_{x}^{2}}=\frac{1}{2}\left[\omega^{\prime \prime}(k) \cos ^{2} \theta+\frac{\omega^{\prime}(k)}{k} \sin ^{2} \theta\right]$

(the prime denotes differentiation); see that the expected dependence $P=\partial^{2} \omega / 2 \partial k^{2}$, which is familiar from nonlinear optics (Hasegawa, 1989) is obtained for $\theta=0$. The full expression for $P$ is given by Eq. (A20) in the Appendix.

The "nonlinearity coefficient" $Q$, which is due to the carrier wave self-interaction, is given by a lengthy expression, reported in the Appendix.

Both coefficients $P$ and $Q$ are functions of $k, \theta$ and $\beta$ (in addition to $\alpha, \alpha^{\prime}$, for $Q$ ), as expected. The exact general expressions obtained (see in the Appendix) may be "tailor fit" to any given electrostatic plasma wave problem (via the form of the parameters $\alpha, \alpha^{\prime}, \beta$ ), in view of a numerical investigation of the wave's amplitude dynamics (e.g. stability profile, wave localization; see in the following). One thus obtains the (tedious) analytical form of $P$ and $Q$ in terms of intrinsic plasma parameters such as background plasma density, temperature, defect concentration, background species composition (if more than one species is present in the background), and so forth. Also note the approximate expressions obtained in the end of the Appendix, which are valid for large wavelength (i.e. small wavenumber) values.

\section{Modulational (in)stability analysis}

It is known (see e.g. in Hasegawa, 1975, 1989; Remoissenet, 1994) that the evolution of a wave whose amplitude obeys Eq. (14) depends on the coefficient product $P Q$, which may be investigated in terms of the physical parameters involved. To see this, first check that Eq. (14) supports the plane (Stokes') wave solution $\psi=\psi_{0} \exp \left(i Q\left|\psi_{0}\right|^{2} T\right)$; the standard linear analysis consists in perturbing the amplitude by setting: $\hat{\psi}=\hat{\psi}_{0}+\epsilon \hat{\psi}_{1,0} \cos (\tilde{k} X-\tilde{\omega} T)$ (the perturbation wavenumber $\hat{k}$ and the frequency $\hat{\omega}$ should be distinguished from their carrier wave homologue quantities, denoted by $k$ and $\omega$ ). One thus obtains the (perturbation) dispersion relation:

$\tilde{\omega}^{2}=P \tilde{k}^{2}\left(P \tilde{k}^{2}-2 Q\left|\hat{\psi}_{1,0}\right|^{2}\right)$.

One immediately sees that if $P Q>0$, the amplitude $\psi$ is "unstable" for $\tilde{k}<\sqrt{2 Q / P}\left|\hat{\psi}_{1,0}\right|$; i.e. for perturbation wavelengths larger than a critical value. If $P Q<0$, the amplitude $\psi$ will be "stable" to external perturbations. This "modulational instability" mechanism is tantamount to the wellknown "Benjamin-Feir" instability, in hydrodynamics, also long-known as an energy localization mechanism in solid state physics and nonlinear optics (Hasegawa, 1989; Infeld, 1990; Remoissenet, 1994).

This type of analysis allows for a numerical investigation of the stability profile in terms of parameters e.g. like wavenumber $k$, perturbation (obliqueness) angle $\alpha$, temperature $T_{\alpha}$, background plasma parameters etc. A few known ES modes have already thus been investigated; see in Sect. 6 below.

\section{Envelope excitations}

It should be pointed out that the evolution Eq. (14) is known to be integrable (Infeld, 1990; Remoissenet, 1994). Its localized solutions, which can be rigorously obtained via the tedious Inverse Scattering Transform method, are properly speaking "solitons", in the sense that they satisfy an infinity of conservation laws; they have been shown analytically (and confirmed numerically) to survive collisions between one another and also exhibit a robust behaviour against external perturbations.

The modulated (electrostatic potential) wave finally resulting from the above analysis is of the form ${ }^{7}$

$\phi_{1}^{(1)}=\epsilon \hat{\psi}_{0} \cos (\mathbf{k r}-\omega t+\Theta)+\mathcal{O}\left(\epsilon^{2}\right)$.

\footnotetext{
${ }^{7}$ In fact, the potential correction amplitude here is $\hat{\psi}_{0}=2 \psi_{0}$, from Euler's formula: $e^{i x}+e^{-i x}=2 \cos x \quad(x \in \Re)$.
} 
Note that once the potential correction $\phi_{1}^{(1)}$ is determined, density, velocity and pressure corrections follow from Eq. (11). The slowly varying amplitude $\psi_{0}(X, T)$ and phase correction $\Theta(X, T)$ (both real functions of $\{X, T\}$; see in Fedele and Schamel, 2002a; Fedele et al., 2002b for details) are determined by (solving) Eq. (14) for

$\psi=\psi_{0} \exp (i \Theta)$.

The different types of solution thus obtained are summarized in the following.

\subsection{Bright-type envelope solitons}

For "positive" $P Q$, the carrier wave is modulationally "unstable"; it may either "collapse", due to (possibly random) external perturbations, or lead to the formation of "bright" envelope modulated wavepackets, i.e. localized envelope "pulses" confining the carrier (see Fig. 4), given by (Fedele and Schamel, 2002a; Fedele et al., 2002b) ${ }^{8}$

$$
\begin{aligned}
\psi_{0} & =\left(\tilde{\psi}_{0}\right)^{1 / 2} \operatorname{sech}\left(\frac{X-v_{e} T}{L}\right), \\
\Theta & =\frac{1}{2 P}\left[v_{e} X+\left(\Omega-\frac{v_{e}^{2}}{2}\right) T\right],
\end{aligned}
$$

where $v_{e}$ is the envelope velocity; $L$ and $\Omega$ represent the pulse's spatial width and oscillation frequency (at rest), respectively. We note that $L$ and $\tilde{\psi}_{0}$ satisfy $L \tilde{\psi}_{0}=$ $(2 P / Q)^{1 / 2}=$ const. (in contrast with KdV solitons (Remoissenet, 1994), where $L^{2} \tilde{\psi}_{0}=$ const. instead). Also, the amplitude $\psi_{0}$ is independent of the pulse (envelope) velocity $v_{e}$ here.

It may be pointed out that, although the bright (envelope) soliton phase profile remains constant as it propagates (see Fig. 6), its phase bears a (slow) space and time dependence, thus allowing for a slight deformation of the wave packet internal structure during propagation; see e.g. Fig. 6, where this effect is clearly pointed out.

\subsection{Black-type envelope solitons}

For $P Q<0$, the carrier wave is modulationally "stable" and may propagate as a "dark" ("black" or "grey") envelope wavepackets, i.e. a propagating localized "hole" (a "void") amidst a uniform wave energy region. The exact expression for "dark" envelopes reads (Fedele and Schamel, 2002a; Fedele et al., 2002b) ${ }^{8}$ :

$$
\begin{aligned}
& \psi_{0}=\psi_{0}^{\prime}\left|\tanh \left(\frac{X-v_{e} T}{L^{\prime}}\right)\right|, \\
& \Theta=\frac{1}{2 P}\left[v_{e} X+\left(2 P Q \psi_{0}^{\prime}{ }_{0}^{2}-\frac{v_{e}^{2}}{2}\right) T\right]
\end{aligned}
$$

(see Fig. 7a); again, $L^{\prime} \psi^{\prime}{ }_{0}=(2|P / Q|)^{1 / 2}$ (=cst.).

\footnotetext{
${ }^{8}$ These expressions are readily obtained from (Fedele and Schamel, 2002a; Fedele et al., 2002b), by shifting the variables therein to our notation as: $x \rightarrow X, s \rightarrow T, \rho_{m} \rightarrow \rho_{0}, \alpha \rightarrow 2 P$, $q_{0} \rightarrow-2 P Q, \Delta \rightarrow L, E \rightarrow \Omega, V_{0} \rightarrow u$.
}

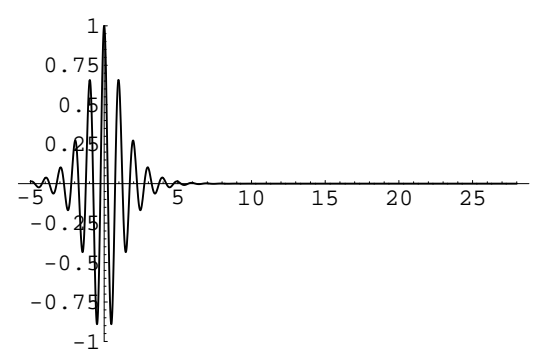

(a)

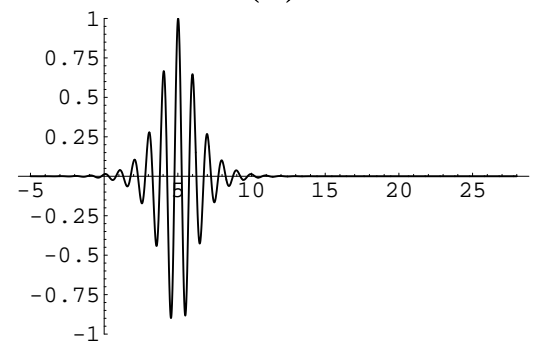

(b)

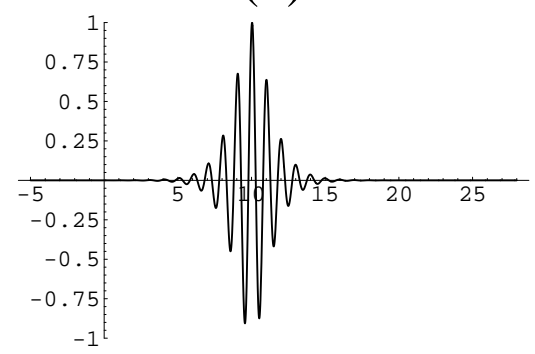

(c)

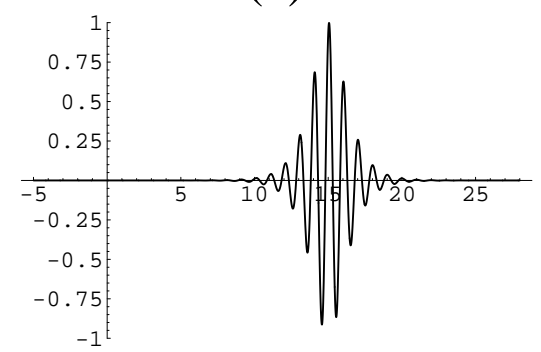

(d)

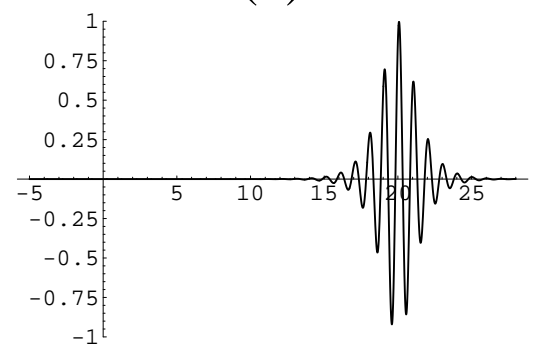

(e)

Fig. 5. Bright envelope soliton propagation, at different times $t_{1}<$ $\cdots<t_{5}$ (arbitrary parameter values): cf. the structures encountered in satellite observations, e.g. see Fig. 3. 


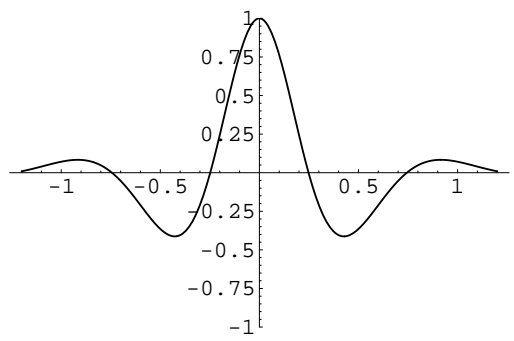

(a)

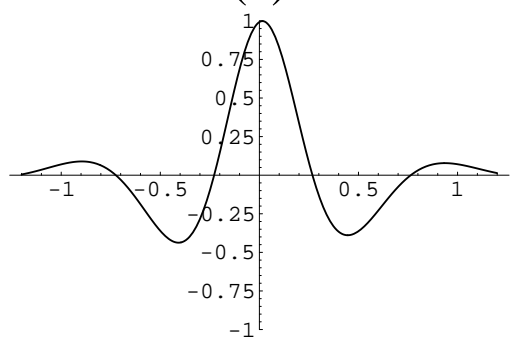

(b)

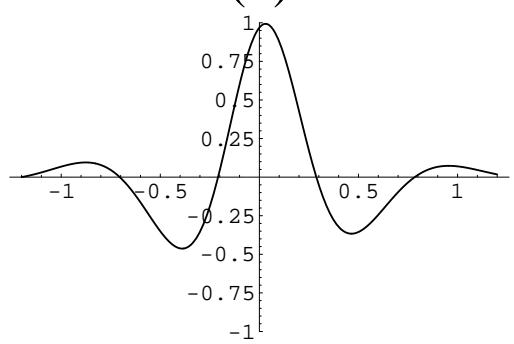

(c)

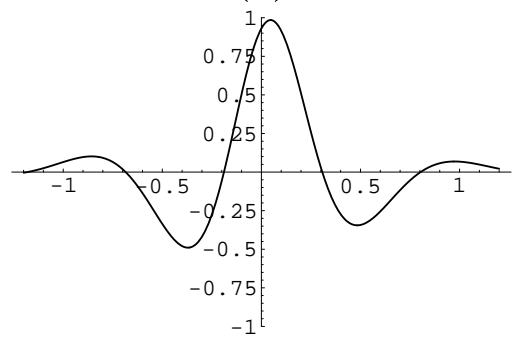

(d)

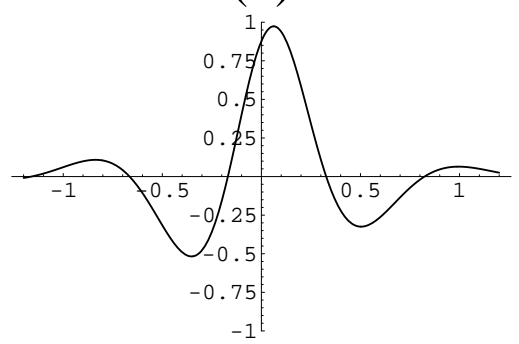

(e)

Fig. 6. Bright envelope soliton profile (centered), at different times $t_{1}<\cdots<t_{5}$ (arbitrary parameter values). Contrary to the previous figure, the envelope width here is comparable in order of magnitude to the carrier wavelength. Notice the variation in the localized packet's internal structure, due to the (slow) phase variation in time.

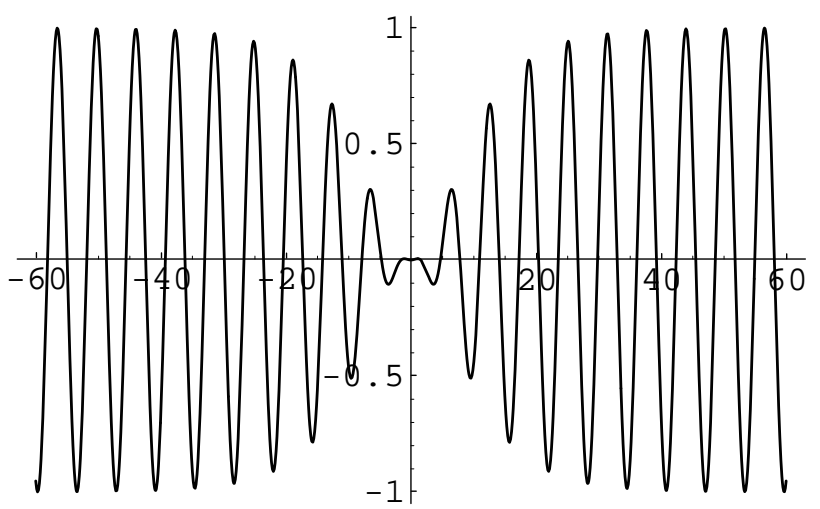

(a)

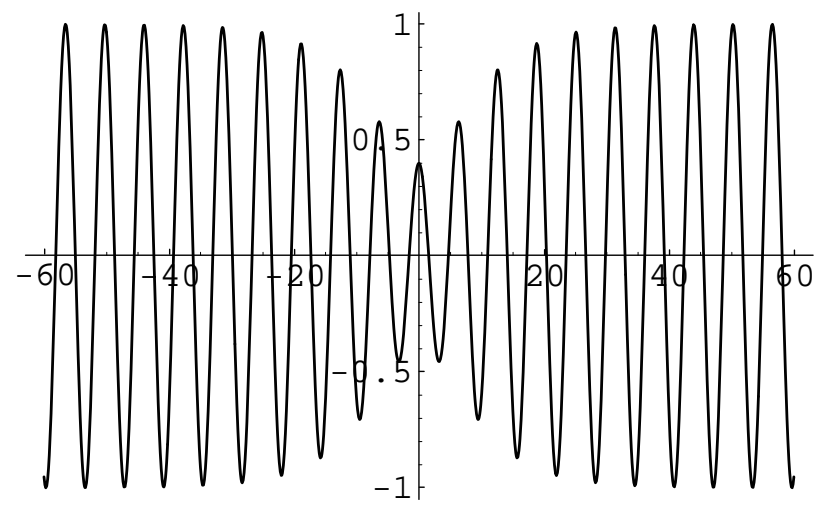

(b)

Fig. 7. "Dark"-type modulated wavepackets (for $P Q<0$ ) of (a) "black" and (b) "grey" kind. See that the amplitude never reaches zero in the latter case.

\subsection{Grey-type envelope solitons}

The "grey"-type envelope (also obtained for $P Q<0$ ) is given by (Fedele and Schamel, 2002a; Fedele et al., 2002b) ${ }^{8}$ :

$\psi_{0}=\psi^{\prime \prime}{ }_{0}\left[1-d^{2} \operatorname{sech}^{2}\left(\frac{X-v_{e} T}{L^{\prime \prime}}\right)\right]^{1 / 2}$

and

$$
\begin{gathered}
\Theta=\frac{1}{2 P}\left[V_{0} X-\left(\frac{1}{2} V_{0}^{2}-2 P Q \psi_{0}^{\prime \prime 2}\right) T+\Theta_{0}\right] \\
-S \sin ^{-1} \frac{d \tanh \left(\frac{X-v_{e} T}{L^{\prime \prime}}\right)}{\left[1-d^{2} \operatorname{sech}^{2}\left(\frac{X-v_{e} T}{L^{\prime \prime}}\right)\right]^{1 / 2}} .
\end{gathered}
$$

Here $\Theta_{0}$ is a constant phase; $S$ denotes the product $S=\operatorname{sign}(P) \times \operatorname{sign}\left(v_{e}-V_{0}\right)$. The pulse width $L^{\prime \prime}=$ $(|P / Q|)^{1 / 2} /\left(d \psi^{\prime \prime}{ }_{0}\right)$ now also depends on the real parameter $d$, given by:

$d^{2}=1+\left(v_{e}-V_{0}\right)^{2} /\left(2 P Q \psi_{0}^{\prime \prime 2}\right) \leq 1$. 


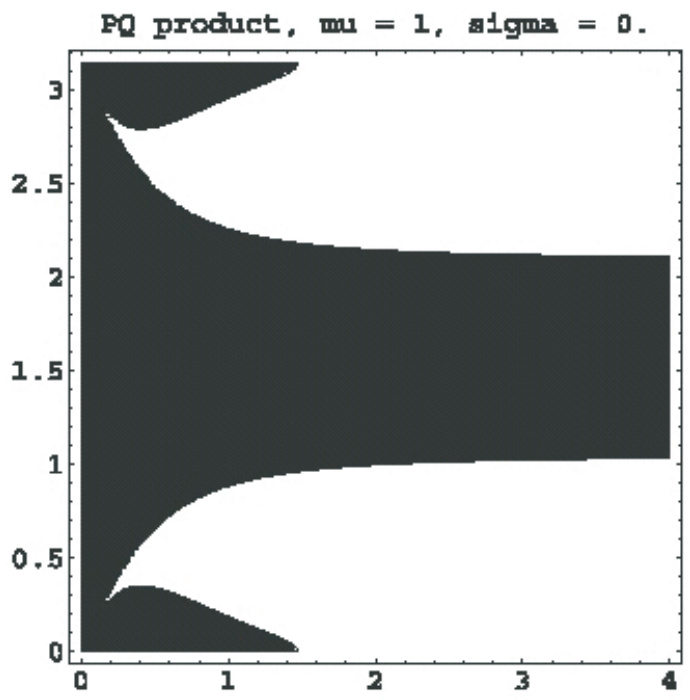

(a)

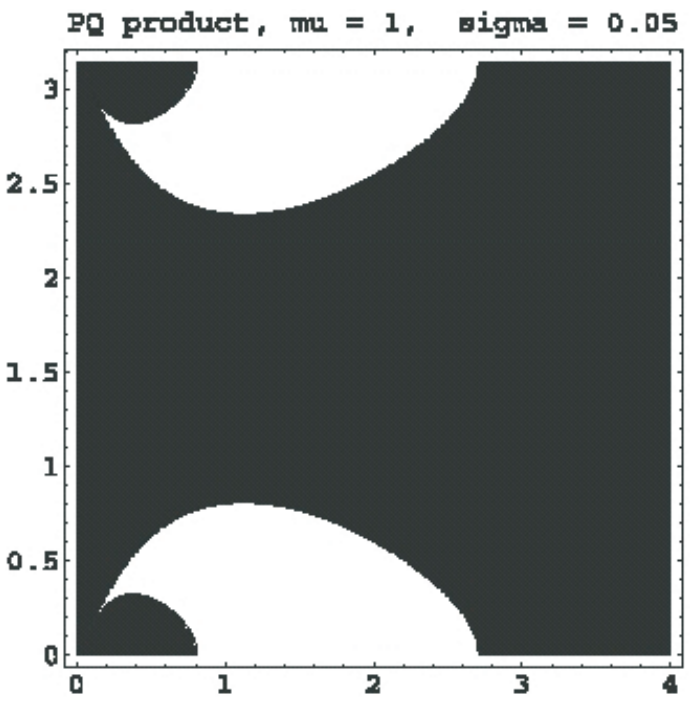

(b)

Fig. 8. The region of positive (negative) values of the product $P Q$, i.e. related to modulational instability (stability), are depicted in white (black), in the "wavenumber" $k$ (horizontal axis) - "modulation angle" $\alpha$ (vertical axis) plane. These plots refer to "ion-acoustic waves": (a) $\sigma=0$ (cold model); (b) $\sigma=0.05$ (warm model) (reprinted from Kourakis and Shukla, 2004b).

The (real) velocity parameter $V_{0}=$ const. satisfies (Fedele and Schamel, 2002a; Fedele et al., 2002b) ${ }^{8}$ :

$V_{0}-\sqrt{2|P Q| \psi_{0}^{\prime \prime 2}} \leq v_{e} \leq V_{0}+\sqrt{2|P Q| \psi_{0}^{\prime \prime 2}}$

For $d=1$ (thus $V_{0}=v_{e}$ ), one recovers the "dark" envelope soliton.

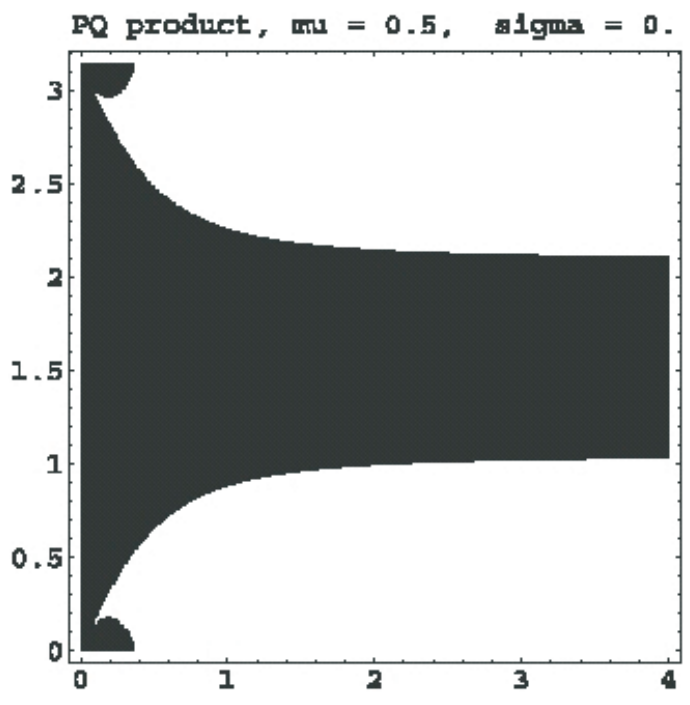

(a)

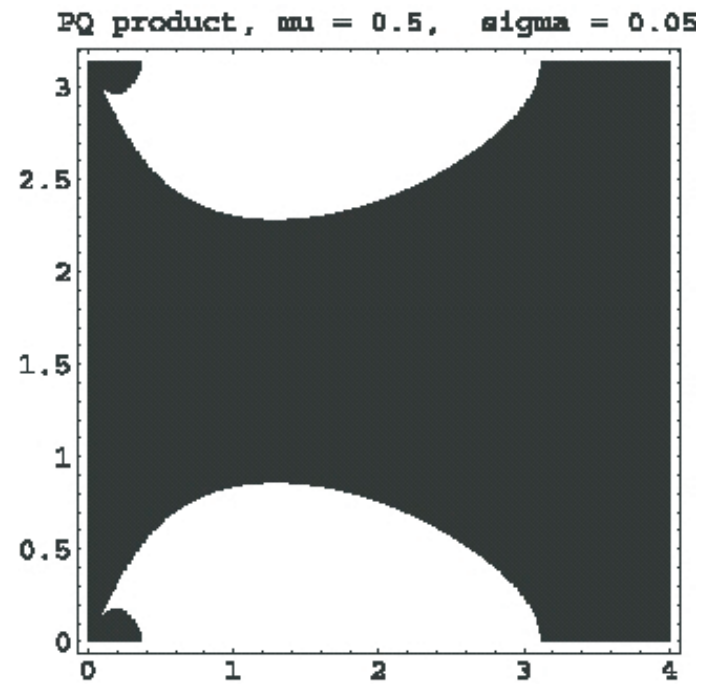

(b)

Fig. 9. Similar to Fig. 8, but for "dust-ion acoustic waves" (see in the text): (a) $\sigma=0$ (cold model); (b) $\sigma=0.05$ (warm model). We have considered a "negative" dust charge: $\delta=q_{d, 0} / q_{i, 0}=0.5$ (i.e. $\left.\mu=n_{e, 0} /\left(Z_{i} n_{i, 0}\right)=0.5\right)$. The dust presence strongly modifies the stability profile, enhancing instability here (reprinted from Kourakis and Shukla, 2004b).

\section{Explicit examples - known plasma modes}

The theory presented here has been recently applied in a variety of plasma modes, for instance: ion acoustic waves (Kourakis and Shukla, 2003a, 2004b) and electron-acoustic waves (Kourakis and Shukla, 2004d). Some previous results regarding parallel modulation were thus generalized to account for oblique modulation (with respect to the propagation direction). In the following, we shall briefly summarize some of these results. 


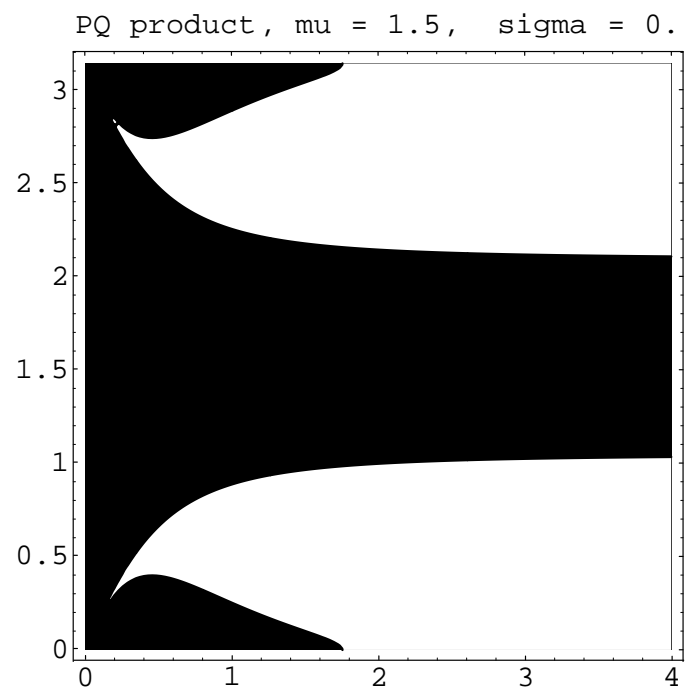

(a)

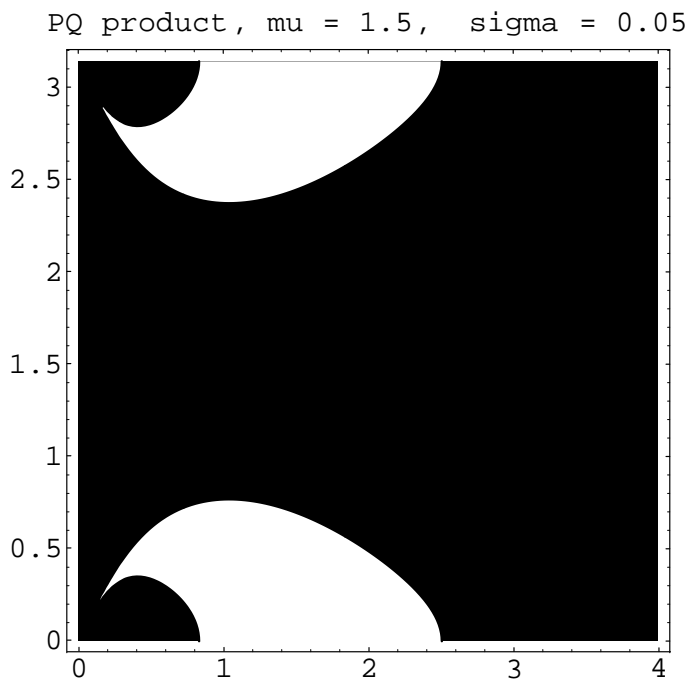

(b)

Fig. 10. Similar to Fig. 8, but for dust-ion acoustic waves in the presence of a "positive" dust charge: (a) $\sigma=0$ (cold model); (b) $\sigma=0.05$ (warm model). We have taken $\delta=q_{d, 0} / q_{i, 0}=0.5$ (i.e. $\left.\mu=n_{e, 0} /\left(Z_{i} n_{i, 0}\right)=1.5\right)$. The positive dust charge seems to favor stability (cf. to the previous figure) (reprinted from Kourakis and Shukla, 2004b).

\subsection{Ion-acoustic waves}

The main result of previous studies of the parallel modulation of ion-acoustic waves, namely the existence of a critical wavenumber, say $k_{c r}$, above which the product $P Q$ becomes positive (i.e. instability may set in), is reproduced via the formalism presented here.

\subsubsection{Obliquity effects}

Allowing for obliquity (by an angle $\theta$ ) between the modulation and propagation directions (cf. the early study by Kako and Hasegawa, 1976) was shown to modify the wave's stability profile rather dramatically: the value of $k_{c r}$ reduces for small values of $\theta$ (say, up to $0.4 \mathrm{rad}$, roughly) and then increases to infinity (prescribing stability) for higher $\theta$; IA waves are globally stable to trasverse modulation (for $\theta=$ $\pi / 2$ ): observe the black region in Fig. 8 a.

\subsubsection{Thermal effects}

Furthermore, allowing for a finite ion temperature ("warm" model, i.e. $\sigma \neq 0$; see Eqs. (4)-(6) above), one witnesses a dramatic modification of the stability profile, mainly via the appearance of a second wavenumber threshold, beyond which stability is recovered: short carrier wavelengths are stable in this "warm" description (cf. Figs. 8a and 8b), as first suggested by Chhabra and Sharma (1986).

\subsection{Dust effects}

The oblique modulation of the "dust-ion acoustic" and dustacoustic ES plasma modes (Shukla and Mamun, 2002) was studied in (Amin et al., 1998; Kourakis and Shukla, 2003a, 2004b) and (Tang and Xue, 2003; Kourakis and Shukla, 2004a), respectively. The generic profile outlined above was here also recovered.

The presence of negative dust particulates was shown to modify the IA wave stability profile, by slightly favoring instability. In the case of positive dust, on the other side, stability was slightly enhanced instead. These results are depicted in Figs. 9 and 10 (to be compared to Fig. 8). A similar qualitative behaviour was witnessed for the DA mode (Tang and Xue, 2003; Kourakis and Shukla, 2004a).

\subsection{Two-temperature effects}

The co-existence of distinct electron populations, say "hot" and "cold" electrons, which is observed in the boundaries of the Earth's magnetosphere, has been shown to affect the modulation behaviour of ion acoustic (Kourakis and Shukla, 2003b) and electron-acoustic waves (Kourakis and Shukla, 2004d). Some of those results are depicted in Figs. 11 to 12, where the presence of the minority electrons appears to yield an important effect even in regions which would have been stable otherwise. Notice the appearance of new instability regions, even for high values of the modulation angle (lateral modulation)

\section{Discussion and conclusion}

We have studied the nonlinear mechanism of amplitude modulation (due to carrier self-interaction) of electrostatic plasma modes. We have shown that the slow variation of the wave's 


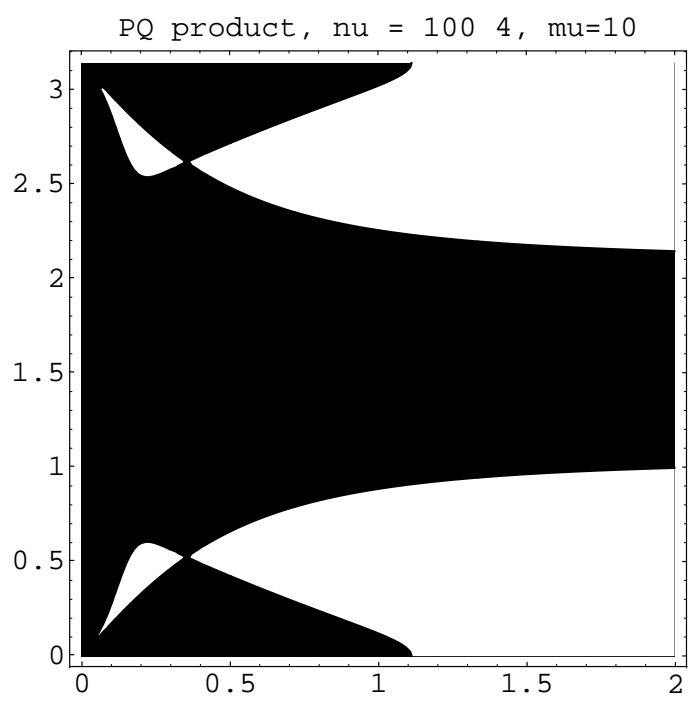

(a)

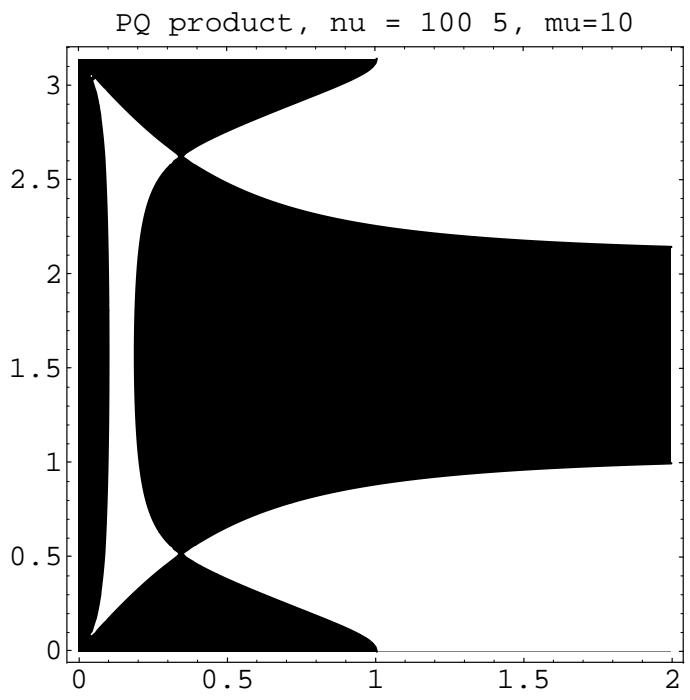

(b)

Fig. 11. Similar to Fig. 8 a $(\sigma=0$, i.e. cold model), but for ion acoustic waves in the presence of two background electron populations ("hot" and "cold" electrons; the former are dominant here). Parameter values are: (a) density ratio $v=n_{h} / n_{c}=100 / 4=$ $25 / 1$, temperature ratio $\mu=T_{h} / T_{c}=10$; (b) $v=n_{h} / n_{c}=$ $100 / 5=20 / 1$, temperature ratio $\mu=T_{h} / T_{c}=10$ (reprinted from Kourakis and Shukla, 2003b).

amplitude in space and time may be modeled via the longestablished multiple scale ("reductive perturbation") method (Taniuti and Yajima, 1969; Asano et al., 1969). One thus obtains explicit conditions for the occurrence of "modulational instability", which is related to wave collapse, or may possibly result in the formation of "localized envelope structures". The criteria thus obtained, in terms of the systems's physical parameters, determine the wave's modulational stability profile and predict the occurrence of localized envelope ex-

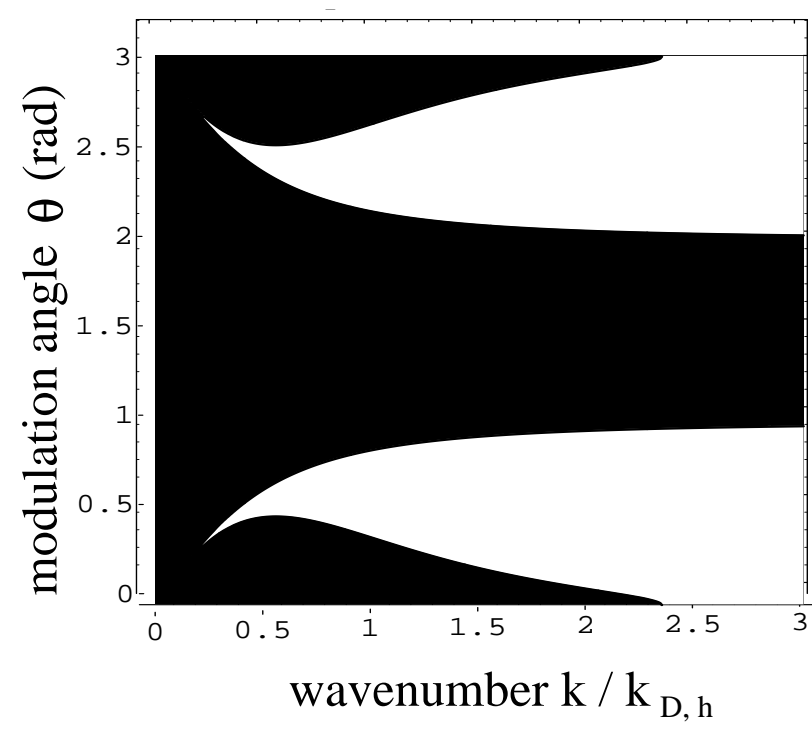

(a)

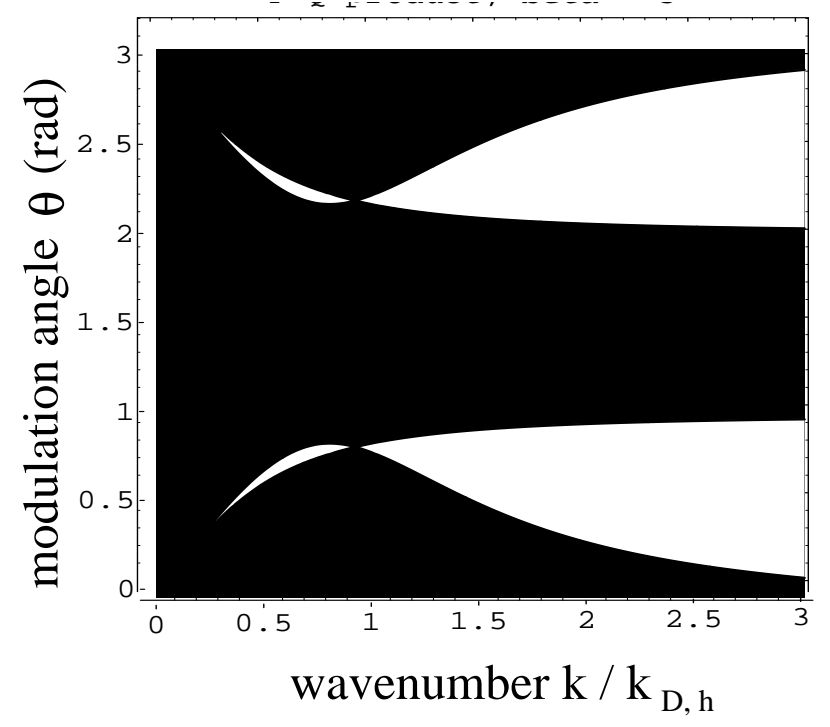

(b)

Fig. 12. A $\{k-\theta\}$-plane plot qualitatively similar to Fig. 8a (for $\sigma=0$, i.e. cold model), but for "electron acoustic waves" in the presence of two background electron populations. Parameter values are: (a) density ratio $v=n_{h} / n_{c}=2 / 1$ (hot electrons dominant); (b) $v=n_{h} / n_{c}=1 / 5$ (cold electrons dominant) (reprinted from Kourakis and Shukla, 2004d).

citations of either bright or dark (black/grey) type. These localized excitations (exact solutions of a nonlinear amplitude evolution equation) provide an efficient model for the electrostatic envelope structures which are abundantly observed during satellite missions and also in laboratory experiments.

It may be mentioned that the results presented herein (and, in particular, the numerical results in the previous Section) should be somehow questioned in wavenumber regions 
where the electrostatic mode considered may be subject to Landau (collisionless) damping. As a matter of fact, this is not an issue in the case of dusty plasma modes (IAW, DAW), where the wave's phase speed lies far from the characteristic thermal velocities of the constituents (Verheest, 2001; Shukla and Mamun, 2002). However, this should be taken into account in the analysis, e.g. with respect to electron-acoustic waves (see the discussion in Kourakis and Shukla, 2004d), since it is known that Landau damping effects (obtained via a kinetic description of ES modes) cannot be predicted by fluid models.

The methodology employed in this article applies in a variety of known electrostatic modes, which can be described by a single fluid model. A generalization of this formalism for plasma modes in the presence of an external magnetic field is on the way and will be reported soon.

\section{Appendix A Perturbative analysis - details}

A1 Harmonic amplitude evolution equations

By substituting into Eqs. (4)-(6) and (10) and isolating distinct orders in $\epsilon$, we obtain the $n$ th-order reduced equations

$$
\begin{aligned}
& -i l \omega n_{l}^{(n)}+i l \mathbf{k} \cdot \mathbf{u}_{l}^{(n)}-\lambda \frac{\partial n_{l}^{(n-1)}}{\partial X} \\
& +\frac{\partial n_{l}^{(n-2)}}{\partial T}+\frac{\partial u_{l, x}^{(n-1)}}{\partial X} \\
& +\sum_{n^{\prime}=1}^{\infty} \sum_{l^{\prime}=-\infty}^{\infty}\left[i l \mathbf{k} \cdot \mathbf{u}_{l-l^{\prime}}^{\left(n-n^{\prime}\right)} n_{l^{\prime}}^{\left(n^{\prime}\right)}\right. \\
& \left.+\frac{\partial}{\partial X}\left(n_{l^{\prime}}^{\left(n^{\prime}\right)} u_{\left(l-l^{\prime}\right), x}^{\left(n-n^{\prime}-1\right)}\right)\right]=0,
\end{aligned}
$$

$$
\begin{aligned}
& -i l \omega \mathbf{u}_{l}^{(n)}+s i l \mathbf{k} \phi_{l}^{(n)}-\lambda \frac{\partial \mathbf{u}_{l}^{(n-1)}}{\partial X}+\frac{\partial \mathbf{u}_{l}^{(n-2)}}{\partial T} \\
& +s \frac{\partial \phi_{l}^{(n-1)}}{\partial X} \hat{x} \\
& +\sum_{n^{\prime}=1}^{\infty} \sum_{l^{\prime}=-\infty}^{\infty}\left[i l^{\prime} \mathbf{k} \cdot \mathbf{u}_{l-l^{\prime}}^{\left(n-n^{\prime}\right)} \mathbf{u}_{l^{\prime}}^{\left(n^{\prime}\right)}+u_{\left(l-l^{\prime}\right), x}^{\left(n-n^{\prime}-1\right)} \frac{\partial \mathbf{u}_{l^{\prime}}^{\left(n^{\prime}\right)}}{\partial X}\right] \\
& +\sigma\left(i l p_{l}^{(n)} \mathbf{k}+\frac{\partial p_{l}^{(n-1)}}{\partial X} \hat{x}\right) \\
& +\sum_{n^{\prime}=1}^{\infty} \sum_{l^{\prime}=-\infty}^{\infty} n_{\left(l-l^{\prime}\right)}^{\left(n-n^{\prime}\right)}\left\{-i l^{\prime} \omega \mathbf{u}_{l^{\prime}}^{\left(n^{\prime}\right)}+s i l^{\prime} \mathbf{k} \phi_{l^{\prime}}^{\left(n^{\prime}\right)}\right. \\
& -\lambda \frac{\partial \mathbf{u}_{l^{\prime}}^{\left(n^{\prime}-1\right)}}{\partial X}+\frac{\partial \mathbf{u}_{l^{\prime}}^{\left(n^{\prime}-2\right)}}{\partial T}+s \frac{\partial \phi_{l^{\prime}}^{\left(n^{\prime}-1\right)}}{\partial X} \hat{x} \\
& +\sum_{n^{\prime \prime}=1}^{\infty} \sum_{l^{\prime \prime}=-\infty}^{\infty}\left[i l^{\prime \prime} \mathbf{k} \cdot \mathbf{u}_{l^{\prime}-l^{\prime \prime}}^{\left(n^{\prime}-n^{\prime \prime}\right)} \mathbf{u}_{l^{\prime \prime}}^{\left(n^{\prime \prime}\right)}\right. \\
& \left.\left.+u_{\left(l^{\prime}-l^{\prime \prime}\right), x}^{\left(n^{\prime}-n^{\prime \prime}-1\right)} \frac{\partial \mathbf{u}_{l^{\prime \prime}}^{\left(n^{\prime \prime}\right)}}{\partial X}\right]\right\}=0,
\end{aligned}
$$

$$
\begin{aligned}
& -i l \omega p_{l}^{(n)}+i l \gamma \mathbf{k} \cdot \mathbf{u}_{l}^{(n)}-\lambda \frac{\partial p_{l}^{(n-1)}}{\partial X} \\
& +\frac{\partial p_{l}^{(n-2)}}{\partial T}+\gamma \frac{\partial u_{l, x}^{(n-1)}}{\partial X} \\
& +\gamma \sum_{n^{\prime}=1}^{\infty} \sum_{l^{\prime}=-\infty}^{\infty} p_{l-l^{\prime}}^{\left(n-n^{\prime}\right)}\left(i l^{\prime} \mathbf{k} \cdot \mathbf{u}_{l^{\prime}}^{\left(n^{\prime}\right)}+\frac{\partial u_{l^{\prime}, x}^{\left(n^{\prime}-1\right)}}{\partial X}\right) \\
& \left.+\sum_{n^{\prime}=1}^{\infty} \sum_{l^{\prime}=-\infty}^{\infty}\right) \\
& \left(i l^{\prime} \mathbf{k} \cdot \mathbf{u}_{l-l^{\prime}}^{\left(n-n^{\prime}\right)} p_{l^{\prime}}^{\left(n^{\prime}\right)}+\frac{\partial u_{l^{\prime}}^{\left(n^{\prime}-1\right)}}{\partial X} u_{\left(l-l^{\prime}\right), x}^{\left(n-n^{\prime}\right)}\right)=0,
\end{aligned}
$$

and

$$
\begin{aligned}
& -\left(l^{2} k^{2}+1\right) \phi_{l}^{(n)}+s \beta n_{l}^{(n)} \\
& +2 i l k_{x} \frac{\partial \phi_{l}^{(n-1)}}{\partial X}+\frac{\partial^{2} \phi_{l}^{(n-2)}}{\partial X^{2}} \\
& +\alpha \sum_{n^{\prime}=1}^{\infty} \sum_{l^{\prime}=-\infty}^{\infty} \phi_{l-l^{\prime}}^{\left(n-n^{\prime}\right)} \phi_{l^{\prime}}^{\left(n^{\prime}\right)} \\
& -\alpha^{\prime} \sum_{n^{\prime}, n^{\prime \prime}=1}^{\infty} \sum_{l^{\prime}, l^{\prime \prime}=-\infty}^{\infty} \phi_{l-l^{\prime}-l^{\prime \prime}}^{\left(n-n^{\prime}-n^{\prime \prime}\right)} \phi_{l^{\prime}}^{\left(n^{\prime}\right)} \phi_{l^{\prime \prime}}^{\left(n^{\prime \prime}\right)} \\
& =0 .
\end{aligned}
$$

A2 First order in $\epsilon$ : first harmonics and dispersion relation The first order $(n=2)$ equations read

$$
\begin{aligned}
-i l \omega n_{l}^{(1)}+i l \mathbf{k} \cdot \mathbf{u}_{l}^{(1)} & =0, \\
-i l \omega \mathbf{u}_{l}^{(1)}+s i l \mathbf{k} \phi_{l}^{(1)}+i l \sigma p_{l}^{(1)} \mathbf{k} & =0, \\
-i l \omega p_{l}^{(1)}+i l \gamma \mathbf{k} \cdot \mathbf{u}_{l}^{(1)} & =0,
\end{aligned}
$$

and

$$
-\left(l^{2} k^{2}+1\right) \phi_{l}^{(1)}+s \beta n_{l}^{(1)}=0 .
$$

For $l=1$, these equations determine the first harmonics of the perturbation. The following dispersion relation is obtained

$$
\omega^{2}=\frac{\beta k^{2}}{k^{2}+1}+\gamma \sigma k^{2} .
$$

Restoring dimensions, one may easily check that the standard DAW dispersion relation (Rao et al., 1990; Shukla and Mamun, 2002) is thus exactly recovered:

$$
\begin{aligned}
\omega^{2} & =\omega_{p, d}^{2} \frac{k^{2}}{k^{2}+k_{D}^{2}}+\gamma \frac{k_{B} T_{d}}{m_{d}} k^{2} \\
& =\equiv \frac{c_{D}^{2} k^{2}}{1+k^{2} \lambda_{D_{e f f}^{2}}^{2}}+\gamma v_{t h, d}^{2} k^{2}
\end{aligned}
$$

The first harmonic amplitudes may now be expressed in terms of the first order potential correction $\phi_{1}^{(1)}$; we obtain 
the relations

$$
\begin{aligned}
n_{1}^{(1)} & =s \frac{1+k^{2}}{\beta} \phi_{1}^{(1)} \\
& \equiv c_{1}^{(11)} \phi_{1}^{(1)}, \\
\mathbf{k} \cdot \mathbf{u}_{1}^{(1)} & =\omega n_{1}^{(1)}=s \omega \frac{1+k^{2}}{\beta} \phi_{1}^{(1)} \\
& \equiv c_{2}^{(11)} \phi_{1}^{(1)}, \\
p_{1}^{(1)} & =\gamma n_{1}^{(1)}=\gamma s \frac{1+k^{2}}{\beta} \phi_{1}^{(1)} \\
& \equiv c_{3}^{(11)} \phi_{1}^{(1)}, \\
u_{1, x}^{(1)} & =\frac{\omega}{k} \cos \theta n_{1}^{(1)}=s \frac{1+k^{2}}{\beta} \frac{\omega}{k} \cos \theta \phi_{1}^{(1)} \\
& \equiv c_{5}^{(11)} \phi_{1}^{(1)}, \\
u_{1, y}^{(1)} & =\frac{\omega}{k} \sin \theta n_{1}^{(1)}=s \frac{1+k^{2}}{\beta} \frac{\omega}{k} \sin \theta \phi_{1}^{(1)},
\end{aligned}
$$

retaining, for later use, the (obvious) definitions of the coefficients $c_{j}^{(11)}(j=1, \ldots, 5)$ relating the state variables to the 1st-order potential correction $\phi_{1}^{(1)}$ (so $\left.c_{4}^{(11)}=1\right)$.

A3 Second order in $\epsilon$ : group velocity, 0th and 2nd harmonics

The second order $(n=2)$ equations for the first harmonics provide the compatibility condition: $\lambda=v_{g}(k)=\frac{\partial \omega}{\partial k_{x}}=$ $\omega^{\prime}(k) \cos \theta=\frac{k}{\omega}\left[\frac{1}{\left(1+k^{2}\right)^{2}}+\gamma \sigma\right] \cos \theta$; the group velocity $v_{g}$ can be cast in the form

$v_{g}(k)=\frac{\omega^{3}}{k^{3}} \frac{\beta+\sigma \gamma\left(1+k^{2}\right)^{2}}{\left[\beta+\sigma \gamma\left(1+k^{2}\right)\right]^{2}} \cos \theta \equiv \frac{\omega^{3}}{\beta k^{3}} \nu_{1} \cos \theta$,

where we have denoted

$\nu_{1}=\beta \frac{\beta+\sigma \gamma\left(1+k^{2}\right)^{2}}{\left[\beta+\sigma \gamma\left(1+k^{2}\right)\right]^{2}}$.

Note that $\nu_{1} \rightarrow 1$ in the limit $\sigma \rightarrow 0$, recovering exactly Eq. (43) in (Amin et al., 1998).

The 2nd-order corrections to the first harmonic amplitudes are now given by

$$
\begin{aligned}
n_{1}^{(2)} & =i s \frac{1}{\beta}\left[\tilde{A}\left(1+k^{2}\right)-2 k \cos \theta\right] \frac{\partial \phi_{1}^{(1)}}{\partial X} \\
& \equiv i c_{1}^{(21)} \frac{\partial \phi_{1}^{(1)}}{\partial X}, \\
\mathbf{k} \cdot \mathbf{u}_{1}^{(2)} & =\omega n_{1}^{(2)}-s \frac{1}{\beta}\left(1+k^{2}\right)\left(v_{g}-\frac{\omega}{k} \cos \theta\right) \frac{\partial \phi_{1}^{(1)}}{\partial X} \\
& \equiv i c_{2}^{(21)} \frac{\partial \phi_{1}^{(1)}}{\partial X}, \\
p_{1}^{(2)} & =\gamma n_{1}^{(2)} \\
& \equiv i c_{3}^{(21)} \frac{\partial \phi_{1}^{(1)}}{\partial X}, \\
\phi_{1}^{(2)} & =i \tilde{A} \frac{\partial \phi_{1}^{(1)}}{\partial X},
\end{aligned}
$$

and

$$
\begin{aligned}
& u_{1, x}^{(2)}= i s \frac{1}{\omega}\left[-1-2 \frac{\gamma}{\beta} \sigma k^{2} \cos ^{2} \theta\right. \\
&\left.+\left(v_{g} \frac{\omega}{k} \cos \theta-\sigma \gamma\right) \frac{1+k^{2}}{\beta}\right] \frac{\partial \phi_{1}^{(1)}}{\partial X}, \\
& \equiv i c_{5}^{(21)} \frac{\partial \phi_{1}^{(1)}}{\partial X} .
\end{aligned}
$$

The choice of the value of $\tilde{A}$ is arbitrary; we shall take $\tilde{A}=0$.

The equations for $n=2, l=2$ provide the amplitudes of the second order harmonics, which are found to be proportional to the square of the corresponding $S_{1}^{(1)}$ elements e.g. in terms of $\phi_{1}^{(1)}$

$$
\begin{aligned}
n_{2}^{(2)}= & {\left[\frac{1}{\omega} A+\frac{\left(1+k^{2}\right)^{2}}{\beta^{2}}\right] \equiv c_{1}^{(22)} \phi_{1}^{(1)^{2}}, } \\
\mathbf{k} \cdot \mathbf{u}_{2}^{(2)}= & \frac{\left(1+k^{2}\right) \omega}{6 \beta^{3} k^{2}}\left[2 s \alpha \beta^{2}+3 \beta\left(1+k^{2}\right)\left(1+2 k^{2}\right)\right. \\
& \left.+2 \gamma^{2} \sigma\left(1+k^{2}\right)^{2}\left(1+4 k^{2}\right)\right] \phi_{1}^{(1)^{2}} \\
\equiv & A \phi_{1}^{(1)^{2}}=c_{2}^{(22)} \phi_{1}^{(1)^{2}}, \\
p_{2}^{(2)}= & \gamma\left[\frac{1}{\omega} A+\gamma \frac{\left(1+k^{2}\right)^{2}}{\beta^{2}}\right] \equiv c_{3}^{(22)} \phi_{1}^{(1)^{2}},
\end{aligned}
$$

and

$$
\begin{aligned}
\phi_{2}^{(2)} & =\frac{1}{4 k^{2}+1}\left\{s \beta\left[\frac{1}{\omega} A+\frac{\left(1+k^{2}\right)^{2}}{\beta^{2}}\right]+\alpha\right\} \phi_{1}^{(1)^{2}} \\
& \equiv c_{4}^{(22)} \phi_{1}^{(1)}{ }^{2} .
\end{aligned}
$$

Notice that these expressions are "isotropic" i.e. independent of the value of $\theta$.

The nonlinear self-interaction of the carrier wave also results in the creation of a zeroth harmonic, in this order; its strength is analytically determined by taking into account the $l=0$ component of the three first third-order reduced equations (i.e. Eqs. (A1)-(A3) for $n=3, l=0$ ) together with the corresponding fourth 2 nd-order equation (i.e. Eq. (A4) for $n=2, l=0$ ). The result is conveniently expressed in terms of the square modulus of the $(n=1, l=1)$ quantities, e.g. in terms of $\left|\phi_{1}^{(1)}\right|^{2}=\left(\phi_{1}^{(1)}\right)^{*} \phi_{1}^{(1)}$

$$
\begin{aligned}
n_{0}^{(2)}= & \frac{-1}{\beta+\gamma \sigma-v_{g}^{2}} \frac{1}{\beta}\left[1+2 s \alpha \beta+k^{2}+2 \cos ^{2} \theta\right. \\
& \left.+\gamma \sigma \frac{\left(1+k^{2}\right)^{2}}{\beta}\left(\gamma+2 \cos ^{2} \theta-1\right)\right]\left|\phi_{1}^{(1)}\right|^{2} \\
\equiv & B\left|\phi_{1}^{(1)}\right|^{2} \\
\equiv & =c_{1}^{(20)}\left|\phi_{1}^{(1)}\right|^{2}, \\
\mathbf{k} \cdot \mathbf{u}_{0}^{(2)}= & \frac{-1}{\beta+\gamma \sigma-v_{g}^{2}} \frac{\cos \theta}{\beta^{2}}\left\{2 \omega(\beta+\gamma \sigma)\left(1+k^{2}\right)^{2}\right. \\
& +k v_{g}\left[\beta\left(1+k^{2}+2 s \alpha \beta\right)\right.
\end{aligned}
$$




$$
\begin{aligned}
& \left.\left.+\sigma \gamma(\gamma-1)\left(1+k^{2}\right)^{2}\right]\right\} \\
\equiv & c_{2}^{(20)}\left|\phi_{1}^{(1)}\right|^{2}, \\
p_{0}^{(2)}= & \left.\gamma\left[B+\frac{1}{\beta^{2}}(\gamma-1)\left(1+k^{2}\right)^{2}\right)\right]\left|\phi_{1}^{(1)}\right|^{2} \\
\equiv & c_{3}^{(20)}\left|\phi_{1}^{(1)}\right|^{2}, \\
\phi_{0}^{(2)}= & (s \beta B+2 \alpha)\left|\phi_{1}^{(1)}\right|^{2} \\
\equiv & c_{4}^{(20)}\left|\phi_{1}^{(1)}\right|^{2},
\end{aligned}
$$

and

$$
\begin{aligned}
u_{0, x}^{(2)} & =\left[v_{g} B-2 \frac{\omega\left(1+k^{2}\right)^{2}}{\beta^{2} k} \cos \theta\right]\left|\phi_{1}^{(1)}\right|^{2} \\
& \equiv c_{5}^{(20)}\left|\phi_{1}^{(1)}\right|^{2} .
\end{aligned}
$$

It is expected, and indeed verified by a tedious yet straightforward calculation, that upon setting $\sigma=0, s=-1$ in expressions (A15) and (A16), one recovers exactly Eqs. (44)-(49) in (Amin et al., 1998) (given Eq. (42) therein).

Notice, for rigor, that for "vanishing obliqueness" i.e. if $\theta \rightarrow 0$, one obviously has $\mathbf{k} \cdot \mathbf{u}_{l}^{(n)} \rightarrow k u_{l}^{(n)}$ (by definition), implying the condition: $c_{2}^{(n l)} \rightarrow k c_{5}^{(n l)}$ (for $\theta \rightarrow 0$ ) which is indeed satisfied for all $n, l$, by the above formulae.

\section{A4 Derivation of the Nonlinear Schrödinger Equation}

Proceeding to the third order in $\epsilon(n=3)$, the equation for $l=1$ yields an explicit compatibility condition to be imposed on the right-hand side of the evolution equations which, given the expressions derived previously, can be cast into the form

$A_{1} \frac{d \psi}{d T}+i A_{2} \frac{d^{2} \psi}{d X^{2}}+i A_{3}|\psi|^{2} \psi=0$,

where $\psi \equiv \phi_{1}^{(1)}$ denotes the amplitude of the first-order electric potential perturbation; coefficients $A_{1,2,3}$ are to be defined. Now, multiplying by $i A_{1}^{-1}$, we obtain the familiar form of the Nonlinear Schrödinger Equation

$i \frac{\partial \psi}{\partial T}+P \frac{\partial^{2} \psi}{\partial X^{2}}+Q|\psi|^{2} \psi=0$.

Recall that the "slow" variables $\{X, T\}$ were defined in Sect. 2.

The "dispersion coefficient" $P=-A_{2} / A_{1}$ is related to the curvature of the dispersion curve as

$P=\frac{1}{2} \frac{\partial^{2} \omega}{\partial k_{x}^{2}}=\frac{1}{2}\left[\omega^{\prime \prime}(k) \cos ^{2} \theta+\omega^{\prime}(k) \frac{\sin ^{2} \theta}{k}\right] ;$

the exact form of $\mathrm{P}$ reads

$P(k)=\frac{1}{\beta} \frac{1}{2 \omega}\left(\frac{\omega}{k}\right)^{4}\left[v_{1}-\left(v_{1}+3 \frac{\nu_{2}}{\beta} \omega^{2}\right) \cos ^{2} \theta\right]$,

where we have defined

$v_{2}=\beta^{3} \frac{3 \beta+\gamma \sigma\left(3-k^{2}\right)\left(1+k^{2}\right)}{3\left[\beta+\gamma \sigma\left(1+k^{2}\right)\right]^{4}}$.
Note that, just like $v_{1}$ defined above, $\nu_{2} \rightarrow 1$ when $\sigma \rightarrow 0$; see that relation (51) in (Amin et al., 1998) is recovered from Eq. (A20) in this case. If, furthermore, we set $\beta=1$ (in addition to $\sigma=0$ ) in all expressions describing our dispersion law i.e. Eqs. (A9), (A12), (A20) above, we obtain, respectively, Eqs. (3), (11), (4) in (Kako and Hasegawa, 1976).

It seems appropriate, here, to point out the qualitative difference between $P$ given in Eq. (A20) as compared to relevant previous expressions: the existence of $\sigma$ may affect the sign of the $P$ coefficient. For instance, taking $\sigma=0$ (i.e. $\left.v_{1}=v_{2}=1\right), P$ is readily seen to be negative for parallel modulation, i.e. setting $\theta=0$; however, for $\sigma \neq 0$ this is no longer the case, since $P$ changes sign at some critical value of $k$ (to see this, study the sign of $v_{2}$ versus $k$ ). Furthermore, a similar remark holds for the effect of an oblique modulation on the sign of $P$; we will come back to this subtle point in the next subsection.

The "nonlinearity coefficient" $Q=-A_{3} / A_{1}$ is due to the carrier wave self-interaction. Distinguishing different contributions, $Q$ can be split into five distinct parts, viz.

$Q=Q_{0}+Q_{1}+Q_{2}+Q_{3}+Q_{4}$,

reflecting the similar structure of $A_{3}$

$$
A_{3}=A_{3}^{(0)}+A_{3}^{(1)}+A_{3}^{(2)}+A_{3}^{(3)}+A_{3}^{(4)} \text {. }
$$

In order to trace the influence of the various parameters, let us define all quantities in full detail. First, $A_{3}^{(0)}$ (as well as $\left.Q_{0}=-A_{3}^{(0)} / A_{1}\right)$ is related to the self-interaction due to the zeroth harmonic, viz.

$$
\begin{aligned}
A_{3}^{(0)}= & -\beta k^{2}\left(c_{1}^{(11)} c_{2}^{(20)}+c_{2}^{(11)} c_{1}^{(20)}\right) \\
& -s \omega 2 \alpha k^{2} c_{4}^{(11)} c_{4}^{(20)}-\omega\left(1+k^{2}\right) c_{2}^{(11)} c_{2}^{(20),}
\end{aligned}
$$

while $A_{3}^{(2)}$ (related to $Q_{2}=-A_{3}^{(2)} / A_{1}$ ) is the analogue quantity due to the second harmonic

$$
\begin{aligned}
A_{3}^{(2)}= & -\beta k^{2}\left(c_{1}^{(11)} c_{2}^{(22)}+c_{2}^{(11)} c_{1}^{(22)}\right) \\
& -s \omega 2 \alpha k^{2} c_{4}^{(11)} c_{4}^{(22)}-\omega\left(1+k^{2}\right) c_{2}^{(11)} c_{2}^{(22)} .
\end{aligned}
$$

All coefficients $c_{j}^{(n l)}$ were defined previously. Now, $Q_{1}=$ $-A_{3}^{(1)} / A_{1}$ is simply the nonlinearity contribution from the cubic term in Eq. (10d) (often omitted in the past)

$A_{3}^{(1)}=+3 s \alpha^{\prime} \omega\left(c_{4}^{(11)}\right)^{3} k^{2}$,

Finally, $A_{3}^{(3)}$ (related to $\left.Q_{3}=-A_{3}^{(3)} / A_{1}\right)$ is the ( $\sigma$-related) result of the third line in Eq. (A2)

$$
\begin{aligned}
A_{3}^{(3)}=-\sigma k^{2}\left(1+k^{2}\right) & {\left[\gamma c_{2}^{(11)}\left(c_{3}^{(20)}-c_{3}^{(22)}\right)\right.} \\
+ & 2 \gamma c_{3}^{(11)} c_{2}^{(22)}+c_{3}^{(11)}\left(c_{2}^{(20)}\right. \\
& \left.\left.-c_{2}^{(22)}\right)+2 c_{2}^{(11)} c_{3}^{(22)}\right]
\end{aligned}
$$


while $A_{3}^{(4)}$ (and $Q_{4}=-A_{3}^{(4)} / A_{1}$ ) is due to the last two lines in Eq. (A2)

$$
\begin{aligned}
A_{3}^{(4)} & =-\omega\left(1+k^{2}\right)\left[\left(\omega c_{2}^{(11)}-s k^{2} c_{4}^{(11)}\right)\left(c_{1}^{(22)}-c_{1}^{(20)}\right)\right. \\
& \left.-2 c_{1}^{(11)}\left(\omega c_{2}^{(22)}-s k^{2} c_{4}^{(22)}\right)+\left(c_{2}^{(11)}\right)^{2} c_{1}^{(11)}\right] . \quad(\mathrm{A} 28)
\end{aligned}
$$

We note that $A_{1}$ is everywhere defined as

$A_{1}=-s \frac{2}{\beta}\left(1+k^{2}\right)^{2} \omega^{2}$,

i.e. by using Eq. (A9)

$A_{1}^{-1}=-s \frac{1}{2 \beta} \frac{1}{\omega^{2}}\left(\frac{\omega^{2}}{k^{2}}-\gamma \sigma\right)^{2}$

(reducing to: $A_{1}^{-1}=-s \frac{1}{2 \beta} \frac{\omega^{2}}{k^{4}}$ for $\sigma=0$ ). Remember that $Q_{3}$ and $Q_{4}$ are plainly absent from the previous results in (Amin et al., 1998) - i.e. for $\sigma=0-$ and so is, in fact, $Q_{1}$.

Substituting from the expressions derived above for the coefficients $c_{j}^{(n l)}$ and re-arranging, we obtain

$$
\begin{aligned}
& Q_{0}=+\frac{1}{2 \omega} \frac{1}{\beta^{2}} \frac{1}{\left(1+k^{2}\right)^{2}} \frac{1}{\beta+\gamma \sigma-v_{g}^{2}} \times \\
& \left\{\beta k ^ { 2 } \left[\beta \left[3+6 k^{2}+4 k^{4}+k^{6}\right.\right.\right. \\
& \left.+2 \alpha \beta\left(s\left(2 k^{2}+3\right)+2 \alpha v_{g}^{2}\right)\right] \\
& +\gamma \sigma\left[(\gamma+1)\left(1+k^{2}\right)^{3}\right. \\
& \left.+2 \alpha \beta\left(-2 \alpha \beta+s \gamma\left(1+k^{2}\right)^{2}\right)\right] \\
& +\left[\beta\left(2+4 k^{2}+3 k^{4}+k^{6}+2 s \alpha \beta\right)\right. \\
& \left.\left.+2 \gamma \sigma\left(1+k^{2}\right)^{2}\left(1+k^{2}+s \alpha \beta\right)\right] \cos 2 \theta\right] \\
& +2\left(1+k^{2}\right)^{4}(\beta+\gamma \sigma) \omega^{2} \cos ^{2} \theta \\
& +k\left(1+k^{2}\right)\left[\beta k^{2}+\omega^{2}\left(1+k^{2}\right)\right] \frac{v_{g}}{\omega} \times \\
& {\left[\beta\left(1+k^{2}+2 s \alpha \beta\right)+\gamma(\gamma-1) \sigma\left(1+k^{2}\right)^{2}\right]} \\
& \times \cos \theta\} \text {, } \\
& Q_{1}=\frac{3 \alpha^{\prime} \beta}{2 \omega} \frac{k^{2}}{\left(1+k^{2}\right)^{2}} \text {, } \\
& Q_{2}=-\frac{1}{12 \beta^{3}} \frac{1}{\omega} \frac{1}{k^{2}\left(1+k^{2}\right)^{2}} \times \\
& \left\{2 \beta k ^ { 2 } \left[5 s \alpha \beta^{2}\left(1+k^{2}\right)^{2}+2 \alpha^{2} \beta^{3}\right.\right. \\
& +2 \gamma^{2} \sigma\left(1+k^{2}\right)^{4}\left(1+4 k^{2}\right) \\
& \left.+\beta\left(1+k^{2}\right)^{3}\left(3+9 k^{2}+2 s \alpha \gamma^{2} \sigma\right)\right] \\
& +\left(1+k^{2}\right)^{3} \omega^{2}\left[\beta\left(3+9 k^{2}+6 k^{4}+2 s \alpha \beta\right)\right. \\
& \left.\left.+2 \gamma^{2} \sigma\left(1+k^{2}\right)^{2}\left(1+4 k^{2}\right)\right]\right\} \text {. }
\end{aligned}
$$

Finally, the coefficients $Q_{3}=-A_{3}^{(3)} / A_{1}$ and $Q_{4}=$ $-A_{3}^{(4)} / A_{1}$ can be directly computed from Eq. (A27)-(A29) above; the lengthy final expressions are omitted here.

Once substituted in Eq. (A22), these expressions provide the final expression for the nonlinearity coefficient $Q$. One may readily check, yet after a tedious calculation, that expressions (A31) and (A33) reduce to Eqs. (53) and (54) in (Amin et al., 1998) for $\sigma=0$. However, the remaining coefficients $Q_{1}, Q_{3}, Q_{4}$ were absent in all previous studies of the DA waves, to the best of our knowledge. Their importance will be discussed in the following. Note that $Q_{1}, Q_{2}$ do not depend on the angle $\theta$.

\section{A5 Behaviour of coefficients for small $k$}

A preliminary result regarding the behaviour (and the sign) of the NLSE coefficients $P$ and $Q$, at least for long wavelengths, may be obtained by considering the limit of small $k \ll 1$ in the above formulae.

The parallel $(\theta=0)$ and oblique $(\theta \neq 0)$ modulation cases have to be distinguished straightaway. For small values of $k$ $(k \ll 1), P$ is negative and varies as

$$
\left.P\right|_{\theta=0} \approx-\frac{3}{2} \frac{\beta}{\sqrt{\beta+\gamma \sigma}} k
$$

in the parallel modulation case (i.e. $\theta=0$ ), thus tending to zero for vanishing $k$, while for $\theta \neq 0, P$ is positive and goes to infinity as

$$
\left.P\right|_{\theta \neq 0} \approx \frac{\sqrt{\beta+\gamma \sigma}}{2 k} \sin ^{2} \theta
$$

for vanishing $k$. Therefore, the slightest deviation by $\theta$ of the amplitude variation direction with respect to the wave propagation direction results in a change in sign of the dispersion coefficient $P$. Given the importance of the coefficient product $P Q$ (to be discussed in the next Section), one may wonder whether this is sufficient for the stability characteristics of the DA wave to change. Let us see what happens with the $Q$ in the limit of small $k$.

For all cases, $Q$ varies as $\sim 1 / k$ for small $k \ll 1$; the exact expression in fact depends on the angle $\theta$. In the general case $(\theta \neq 0)$, the result reads

$$
\begin{aligned}
\left.Q\right|_{\theta \neq 0} \approx & -\frac{1}{12 \beta^{3}} \frac{1}{\sqrt{\beta+\gamma \sigma}}\left[\beta(2 s \alpha \beta+3)+2 \gamma^{2} \sigma\right] \\
& \times[\beta(2 s \alpha \beta+3)+\gamma(\gamma+1) \sigma] \frac{1}{k} .
\end{aligned}
$$

A careful study shows that $Q$ is negative, in fact, for all possible values of the physical parameters of interest (i.e. $\alpha, \beta$, $\gamma, \sigma-$ all positive - "and" $s \pm 1$ ). For vanishing $\theta$, however, the approximate expression for $Q$, yet apparently quite similar, is now "positive", i.e.

$$
\begin{aligned}
\left.Q\right|_{\theta=0} \approx & +\frac{1}{12 \beta^{3}} \frac{1}{\sqrt{\beta+\gamma \sigma}}[\beta(2 s \alpha \beta+3)+2 \gamma \sigma] \\
& \times[\beta(2 s \alpha \beta+3)+\gamma(\gamma+1) \sigma] \frac{1}{k} .
\end{aligned}
$$


Notice, for rigor, that these formulae are in agreement with the (known) case of the parallel-modulated ion-acoustic waves: see Eq. (41) in Shimizu and Ichikawa (1972); as a matter of fact, the factor $1 / 3$ therein is also exactly recovered here upon setting the appropriate parameter values into Eq. (A37).

In conclusion, both coefficients $P$ and $Q$ change sign when switching on "theta". Indeed, obliqueness in modulation is expected to influence the stability profile of the system; this point seems to confirm (and complete) the general qualitative arguments put forward in (Kako and Hasegawa, 1976) for the ion acoustic wave in an electron ion plasma without dust. Nevertheless, at all cases, the product of $P$ and $Q$ is negative for small $k$, ensuring, as we shall see in the following section, stability for long perturbation wavelengths. As a by-product of this analysis, we see that taking into account $Q_{1}, Q_{3}$ and $Q_{4}$ does not seem to influence the dynamics in the low wavenumber $k$ parameter range.

Acknowledgements. This work was supported by the SFB591 (Sonderforschungsbereich) - Universelles Verhalten gleichgewichtsferner Plasmen: Heizung, Transport und Strukturbildung German government Programme, as well as the International Space Science Institute (ISSI), Bern, Switzerland.

I. Kourakis is grateful to Max-Planck-Institut für extraterrestrische Physik (Garching, Germany) for the award of a fellowship (project: "Komplexe Plasmen").

Figure 1 is reprinted from: Santolik, O., Gurnett, D. A., Pickett, J. S., Parrot, M., and Cornilleau-Wehrlin, N.: Spatio-temporal structure of storm-time chorus, J. Geophys. Res., 108, 1278/1-14 (2003); Copyright (2003) American Geophysical Union; reproduced by permission of the American Geophysical Union.

Figure 2 is reprinted from: Pottelette, R., Ergun, R. E., Treumann, R. A., Berthomier, M., Carlson, C. W., McFadden, J. P., and Roth, I.: Modulated electron-acoustic waves in auroral density cavities: FAST observations, Geophys. Res. Lett., 26, 16, 2629-2632, 1999; Copyright (1999) American Geophysical Union; reproduced by permission of the American Geophysical Union.

Figure 3 is reprinted from: Alpert, Ya.: Resonance nature of the magnetosphere, Physics Reports 339, 323-444, 2001; Copyright (2001) Elsevier; reproduced by permission of Elsevier.

Figures $8-10$ is reprinted from: Kourakis, I. and Shukla, P. K.: Finite ion temperature effects on the stability and envelope excitations of dust-ion acoustic waves, European Physical Journal D, 28, 109117, 2004; Copyright (2003) Springer; reproduced by permission of Springer.

Figure 11 is reprinted from: Kourakis, I. and Shukla, P. K.: Ionacoustic waves in a two-electron-temperature plasma: oblique modulation and envelope excitations, Journal of Physics A: Mathematical \& General, 36, 11 901-11 913, 2003; Copyright (2003) Institute of Physics.

Figure 12 is reprinted from: Kourakis, I. and Shukla, P. K.: Electron-acoustic plasma waves: oblique modulation and envelope solitons, Physical Review E, 69, 3, 036411/1-7, 2004; Copyright (2004) American Physical Society.

Edited by: J. F. McKenzie

Reviewed by: two referees

\section{References}

Alpert, Ya.: Resonance nature of the magnetosphere, Physics Reports, 339, 323-444, 2001.

Amin, M. R., Morfill, G. E., and Shukla, P. K.: Modulational instability of dust-acoustic and dust-ion-acoustic waves, Phys. Rev. E, 58, 6517-6523, 1998.

Asano, N., Taniuti, T., and Yajima, N.: Perturbation method for a nonlinear wave modulation II, J. Math. Phys., 10, 2020-2024, 1969.

Bailung, H., and Nakamura, Y.: Observation of modulational instability in a multi-component plasma with negative ions, J. Plasma Phys., 50, 2, 231-242, 1993.

Berthomier, M., Pottelette, R., and Malingre, M.: Solitary waves and weak double layers in a two-electron temperature auroral plasma, J. Geophys. Res., 103, A3, 4261-4270, 1998.

Boström, R.: Characteristics of Solitary Waves and Weak Double Layers in the Magnetospheric Plasma, Phys. Rev. Lett., 61, 82$85,1988$.

Cattell, C. A., Dombeck, J., Wygant, J. R., Hudson, M. K., Mozer, F. S., Temerin, M. A., Peterson, W. K., Kletzing, C. A., Russell, C. T., and Pfaff, R.: Comparisons of Polar satellite observations of solitary wave velocities in the plasma sheet boundary and the high altitude cusp to those in the auroral zone, Geophys. Res. Lett., 26, 425-428, 1999.

Cattell, C. A., Neiman, C., Dombeck, J., Crumley, J., Wygant, J., Kletzing, C. A., Peterson, W. K., Mozer, F. S., and André, M.: Large amplitude solitary waves in and near the Earth's magnetosphere, magnetopause and bow shock: Polar and Cluster observations, Nonlin. Proc. Geophys., 10, 13-26, 2003,

SRef-ID: 1607-7946/npg/2003-10-13.

Chhabra, R. and Sharma, S.: Modulational instability of obliquely modulated ion-acoustic waves in a two-ion plasma, Phys. Fluids, 29, 128-132, 1986.

Chan, V. and Seshadri, S.: Modulational stability of the ion plasma mode, Phys. Fluids, 18, 1294-1298, 1975.

Davydov, A. S.: Solitons in Molecular Systems, Kluwer Academic Publishers, Dordrecht, 1985.

Delory, G. T., Ergun, R. E., Carlson, C. W., Muschietti, L., Chaston, C. C., Peria, W., and McFadden, J. P.: FAST observations of electron distributions within AKR source regions, Geophys. Res. Lett., 25, 12, 2069-2072, 1998.

Durrani, I. R., Murtaza, G., Rahman, H. U., and Azhar, I. A.: Effect of ionic temperature on the modulational instability of ion acoustic waves in a collisionless plasma, Phys. Fluids, 22, 791793, 1979.

Eliasson, B. and Shukla, P. K.: Theoretical and numerical studies of density modulated whistlers, Geophys. Res. Lett., 31, L17802/14, 2004.

Ergun, R. E., Carlson, C. W., McFadden, J. P., Mozer, F. S., Delory, G. T., Peria, W., Chaston, C. C., Temerin, M., Roth, I., Muschietti, L., Elphic, R., Strangeway, R., Pfaff, R., Cattell, C. A., Klumpar, D., Shelley, E., Peterson, W., Moebius, E., and Kistler, L.: FAST satellite observations of large-amplitude solitary structures, Geophys. Res. Lett., 25, 2041-2044, 1998a.

Ergun, R. E., Carlson, C. W., McFadden, J. P., Mozer, F. S., Delory, G. T., Peria, W., Chaston, C. C., Temerin, M., Elphic, R., Strangeway, R., Pfaff, R., Cattell, C. A., Klumpar, D., Shelley, E., Peterson, W., Moebius, E., and Kistler, L.: FAST satellite wave observations in the AKR source region, Geophys. Res. Lett., 25, 2061-2064, 1998b. 
Fedele, R. and Schamel, H.: Solitary waves in the Madelung's Fluid: A Connection between the nonlinear Schrödinger equation and the Korteweg-de Vries equation, Eur. Phys. J. B, 27, 313-320, 2002a.

Fedele, R., Schamel, H., and P. K. Shukla: Solitons in the Madelung's Fluid, Phys. Scripta, T98, 18-23, 2002b.

Franz, J. R., Kintner, P. M., and Pickett, J. S.: POLAR observations of coherent electric field structures, Geophys. Res. Lett., 25, 8, 1277-1280, 1998.

Hasegawa, A.: Stimulated Modulational Instabilities of Plasma Waves, Phys. Rev. A, 1, 6, 1746-1750, 1970.

Hasegawa, A.: Theory and Computer Experiment on Self-Trapping Instability of Plasma Cyclotron Waves, Phys. Fluids, 15, 5, 870 $881,1972$.

Hasegawa, A.: Plasma Instabilities and Nonlinear Effects, SpringerVerlag, Berlin, 1975.

Hasegawa, A.: Optical Solitons in Fibers, Springer-Verlag, 1989.

Infeld, E. and Rowlands, G.: Nonlinear Waves, Solitons and Chaos, Cambridge University Press, Cambridge, United Kingdom, 1990.

Kako, M.: Part III - Applications to the Collisionless Plasma in Fluid Model, B. Nonlinear Modulation of Plasma Waves, Prog. Theor. Phys. Suppl., 55, 1, 120-137, 1974.

M. Kako and A. Hasegawa: Stability of oblique modulation on an ion-acoustic wave, Phys. Fluids, 19, 1967-1969, 1976.

Kakutani, T. and Sugimoto, N.: Krylov-Bogolibov-Mitropolsky method for nonlinear wave modulation, Phys. Fluids, 17, 16171625,1974

Kostrov, A. V., Gushchin, M. E., Korobkov, S. V., and Strikovskii, A. V.: Parametric Transformation of the Amplitude and Frequency of a Whistler Wave in a Magnetoactive Plasma, JETP Lett., 78, 538-541, 2003.

Kourakis, I. and Shukla, P. K.: Modulational instability and localized excitations of dust-ion acoustic waves, Physics of Plasmas, 10, 9, 3459-3470, 2003a.

Kourakis, I. and Shukla, P. K.: Ion-acoustic waves in a twoelectron-temperature plasma: oblique modulation and envelope excitations, J. Phys. A: Math. Gen., 36, 11 901-11 913, $2003 \mathrm{~b}$.

Kourakis, I. and Shukla, P. K.: Oblique amplitude modulation of dust-acoustic plasma waves, Phys. Scripta, 69, 4, 316-327, 2004a.

Kourakis, I. and Shukla, P. K.: Finite ion temperature effects on the stability and envelope excitations of dust-ion acoustic waves, European Physical Journal D, 28, 109-117, 2004b.

Kourakis, I. and Shukla, P. K.: Linear and nonlinear properties of Rao-dust-Alfvén waves in magnetized plasmas, Physics of Plasmas, 11, 3, 958-969, 2004c.

Kourakis, I. and Shukla, P. K.: Electron-acoustic plasma waves: oblique modulation and envelope solitons, Phys. Rev. E, 69, 3, 036411/1-7, 2004d.

Krall, N. A., and Trivelpiece, A. W.: Principles of plasma physics, McGraw - Hill New York, 1973
Luo, Q.-Z., D'Angelo, N., and Merlino, R.: Shock formation in a negative ion plasma, Phys. Plasmas, 5, 9, 2868-2870, 1998.

Matsumoto, H., Kojima, H., Miyatake, T., Omura, Y., Okada, M., Nagano, I., Tsutsui, M.: Electrostatic solitary waves (ESW) in the magnetotail: BEN wave forms observed by GEOTAIL, Geophys. Res. Lett., 21, 25, 2915-2918, 1994.

McFadden, J. P., Carlson, C. W., Ergun, R. E., Mozer, F. S., Muschietti, L., Roth, I., and Moebius, E.: FAST observations of ion solitary waves, J. Geophys. Res., 108, 4, 8018, doi:10.1029/2002JA009485, 2003.

Mishra, M., Chhabra, R., and Sharma, S.: Stability of oblique modulation of ion-acoustic waves in a multicomponent plasma, Phys. Plasmas, 1, 70-75, 1994

Nakamura, Y., Bailung, H., and Shukla, P. K.: Observation of IonAcoustic Shocks in a Dusty Plasma, Phys. Rev. Lett., 83, 8, 1602-1605, 1999.

Nakamura, Y. and Sarma, A.: Observation of ion-acoustic solitary waves in a dusty plasma, Phys. Plasmas, 8, 9, 3921-3926, 2001.

Pottelette, R., Ergun, R. E., Treumann, R. A., Berthomier, M., Carlson, C. W., McFadden, J. P., and Roth, I.: Modulated electronacoustic waves in auroral density cavities: FAST observations, Geophys. Res. Lett., 26, 16, 2629-2632, 1999.

Rao, N. N., Shukla, P. K., and Yu, M. Y.: Dust-acoustic waves in dusty plasmas, Planet. Space Sci., 38, 543-546, 1990.

Remoissenet, M.: Waves Called Solitons, Springer-Verlag, Berlin, 1994.

Santolik, O., Gurnett, D. A., Pickett, J. S., Parrot, M., and Cornilleau-Wehrlin, N.: Spatio-temporal structure of storm-time chorus, J. Geophys. Res., 108, 1278/1-14, 2003.

Shimizu, K. and Ichikawa, H.: Automodulation of Ion Oscillation Modes in Plasma, J. Phys. Soc. Japan, 33, 789-792, 1972.

Shukla, P. K. and Silin, V. P.: Dust Ion-Acoustic Wave, Physica Scripta, 45, 508-508, 1992.

Shukla, P. K. and Mamun, A. A.: Introduction to Dusty Plasma Physics, Institute of Physics Publishing Ltd., Bristol, 2002.

Stix, Th. : Waves in Plasmas, American Institute of Physics, New York, 1992.

Swanson, D. G.: Plasma Waves, Institute of Physics Publishing Ltd., Bristol, 2003.

Tang, R.-A. and Xue, J.-K.: Stability of oblique modulation of dustacoustic waves in a warm dusty plasma with dust charge variation, Phys. Plasmas, 10, 3800-3803, 2003.

Taniuti, T. and Yajima, N.: Perturbation method for a nonlinear wave modulation I, J. Math. Phys., 10, 1369-1372, 1969.

Temerin, M.: Observations of Double Layers and Solitary Waves in the Auroral Plasma, Phys. Rev. Lett., 48, 1175-1179, 1982.

Verheest, F.: Waves in Dusty Space Plasmas, Kluwer Academic Publishers, Dordrecht, 2001.

Watanabe, W.: Self-modulation of a nonlinear ion wave packet, J. Plasma Phys., 17, 487-501, 1977. 